\title{
CD127 imprints functional heterogeneity to diversify monocyte responses in human inflammatory diseases
}

\author{
Bin Zhang ${ }^{1,2,13}$, Yuan Zhang ${ }^{1,2,10,13}$, Lei Xiong ${ }^{3}$, Yuzhe Li ${ }^{4}$, Yunliang Zhang ${ }^{1,2,11}$, Jiuliang Zhao ${ }^{5}$, Hui \\ Jiang ${ }^{5}$, Can Li ${ }^{5,12}$, Yunqi Liu ${ }^{1,2}$, Xindong Liu ${ }^{6}$, Haofei Liu ${ }^{6}$, Yi-Fang Ping ${ }^{6}$, Qiangfeng Cliff Zhang ${ }^{3,7}$, \\ Zheng Zhang ${ }^{8,9}$, Xiu-Wu Bian ${ }^{6 *}$, Yan Zhao ${ }^{5^{*}} \&$ Xiaoyu $\mathrm{Hu}^{1,2,7^{*}}$
}

\section{Affiliations:}

${ }^{1}$ Institute for Immunology and School of Medicine, Tsinghua University, Beijing, China

${ }^{2}$ Beijing Key Laboratory for Immunological Research on Chronic Diseases, Beijing, China

${ }^{3}$ MOE Key Laboratory of Bioinformatics, Beijing Advanced Innovation Center for Structural Biology, Center for Synthetic and Systems Biology, School of Life Sciences, Tsinghua University, Beijing, China

${ }^{4}$ Academy for Advanced Interdisciplinary Studies, Peking University, Beijing, China

${ }^{5}$ Department of Rheumatology, Peking Union Medical College Hospital, Chinese Academy of Medical

Science and Peking Union Medical College, National Clinical Research Center for Dermatologic and Immunologic Diseases (NCRC-DID), Beijing, China

${ }^{6}$ Institute of Pathology, Southwest Hospital, Third Military Medical University (Army Medical University), Chongqing, China

${ }^{7}$ Tsinghua-Peking Center for Life Sciences, Beijing, China

${ }^{8}$ Institute for Hepatology, National Clinical Research Center for Infectious Disease, Shenzhen Third

People's Hospital, Shenzhen, China

${ }^{9}$ The Second Affiliated Hospital, School of Medicine, Southern University of Science and Technology, Shenzhen, China

${ }^{10}$ Current address: Department of Pathology, Immunology and Laboratory Medicine, University of Florida, Gainesville, Florida, USA

${ }^{11}$ Current address: Laboratory of Viral Diseases, National Institute of Allergy and Infectious Diseases, National Institutes of Health, Bethesda, Maryland, USA

${ }^{12}$ Current address: Department of Cardiology, National Center for Cardiovascular Diseases Fuwai Hospital, Chinese Academy of Medical Sciences and Peking Union Medical College, Beijing, China

${ }^{13}$ These authors contributed equally

*Correspondence to: xiaoyuhu@tsinghua.edu.cn, zhaoyan_pumch2002@aliyun.com,bianxiuwu@263.net. 


\section{Abstract:}

33 Studies on human monocytes historically focused on characterization of bulk responses, whereas functional 34 heterogeneity is largely unknown. Here, we identified an inducible population of CD127-expressing human 35 monocytes under inflammatory conditions and named the subset M127. M127 is nearly absent in healthy 36 individuals yet abundantly present in patients with infectious and inflammatory conditions such as COVID3719 and rheumatoid arthritis. Multiple genomic and functional approaches revealed unique gene signatures of 38 M127 and unified anti-inflammatory properties imposed by the CD127-STAT5 axis. M127 expansion 39 correlated with mild COVID-19 disease outcomes. Thereby, we phenotypically and molecularly 40 characterized a human monocyte subset marked by CD127 that retained anti-inflammatory properties within 41 the pro-inflammatory environments, uncovering remarkable functional diversity among monocytes and 42 signifying M127 as a potential therapeutic target for human inflammatory disorders. 


\section{Main Text:}

Human monocytes and macrophages are considered major mediators of inflammation in a plethora of disease settings including infectious diseases such as COVID-19 and chronic inflammatory diseases such as rheumatoid arthritis (RA) ${ }^{1-3}$. During the pathological processes, inflammatory monocytes from peripheral blood origin accumulate at the sites of infection and/or inflammation and produce large quantities of proinflammatory mediators including cytokines and chemokines ${ }^{4}$, exacerbating disease outcomes by promoting the vicious inflammation cycle ${ }^{5,6}$. Under homeostasis, human monocyte heterogeneity has been conventionally defined by bimodal expression of CD14 and CD16 ${ }^{7,8}$. However, understanding of functional heterogeneity of human monocytes under inflammatory conditions is limited, which imposes conceptual and technical barriers of therapeutically targeting human inflammatory diseases ${ }^{9}$, a particularly prominent issue amid the global menace of COVID-19 ${ }^{10}$. Here, through multiomics analyses of human samples including extensive profiling at the single cell level, we defined a subset of human inflammatory monocytes uniquely marked by the expression of CD127 (thus termed M127) that were abundantly present in inflamed tissues of COVID-19 and RA patients. Mechanistic investigations and integrative computational approaches further revealed common molecular and functional features of M127 across multiple inflammatory disease conditions.

CD127, encoded by $I L 7 R$, is generally considered a lymphoid lineage marker that is predominantly expressed and functional on $\mathrm{T}$ cells and innate lymphoid cells ${ }^{11}$. Immunohistochemical analyses of pulmonary autopsy samples revealed minimal CD127 expression in alveoli from individuals who deceased due to non-infectious causes yet robust staining in patients succumbing to SARS-CoV-2 infection (Fig. 1a). Unexpectedly, in SARS-CoV-2 infected lung tissues, CD127 signals appeared in the regions of CD68 positivity (Fig. 1a), implying plausible expression of CD127 in monocytes/macrophages, which was confirmed by co-localization of CD127 and CD68 signals on immunofluorescently stained sections of autopsied COVID-19 lung tissues (Fig. 1b). Monocytes/macrophage (CD14 ${ }^{\text {high }}$ CD68 ${ }^{\text {high }}$ ) expression of CD127 was further corroborated at the transcriptome level by single cell RNA sequencing (scRNA-seq) analyses of bronchoalveolar lavage fluid (BALF) from nine COVID-19 patients with clinical manifestations ranging from mild $(\mathrm{n}=3)$ to severe $(\mathrm{n}=6)$ (Extended Data Fig. 1a). Strikingly, a distinct $I L 7 R^{+}$population (Fig. 1c,d) was revealed to constitute $21 \%$ of BALF monocytes/macrophages (Fig. 1e). Moreover, in contrast to the predicted dominance by lymphoid cells, the majority (64\%) of $I L 7 R^{+}$cells in COVID-19 BALF were of monocyte/macrophage lineage (Fig. 1f).

To validate whether CD127 expression on monocytes/macrophages can be generalized to other human inflammatory conditions such as RA, peripheral blood mononuclear cells (PBMCs) from 16 antiinflammatory treatment naïve RA patients were analyzed for CD127 expression. Compared with the minimal levels of CD127 in CD14 $4^{+}$monocytes from healthy donors, all RA patients examined displayed markedly elevated expression of CD127 on blood monocytes at the protein and mRNA levels (Fig. 1g,h). In RA blood 
monocytes, $I L 7 R$ expression correlated with the expression of a major pathogenic factor, TNF (Fig. $1 \mathrm{~h}, \mathrm{i}){ }^{12}$. To probe CD127 at the primary sites of inflammation, RA synovial tissue scRNA-seq data sets from 18 patients $^{13}$ (Extended Data Fig. 1b) were analyzed for $I L 7 R$ expression, which showed strong positivity in a distinct population (Fig. $1 \mathrm{j}, \mathrm{k}$ ) that represented nearly $22 \%$ of synovial monocytes (Fig. 11). Taken together, expression of CD127 on a subset of inflammatory monocytes/macrophages is likely a hallmark of human inflammatory conditions testified in multiple disease settings (COVID-19 and RA) and multiple tissues (infected lungs, peripheral blood and inflamed joints).

In order to pursue in-depth investigation of $\mathrm{CD} 127^{+}$monocytes/macrophages, we wished to recapitulate such phenotypes in vitro using infectious and inflammatory stimuli. Stimulation of human PBMCs from healthy donors with toll-like receptor (TLR) ligands led to drastic increase of monocytic CD127 at the protein and mRNA levels, with the exception of TLR3 agonist poly(I:C) (Fig. 2a,b). Upregulation of CD127 was dynamic, peaking around $6 \mathrm{~h}$ post LPS stimulation (Fig. 2c,d), dependent on canonical TLR signaling modules such as IKK and p38 (Extended Data Fig. 2a-e), and observed in all three currently defined human monocyte subpopulations (Fig. 2e and Extended Data Fig. 2f,g). In contrast to human monocytes, LPS failed to upregulate CD127 in murine peripheral blood monocytes (Extended Data Fig. 3a-c) and macrophages from multiple tissue sources (data not shown), suggesting human-specific nature of CD127 induction. In addition to TLR stimulations that mimicked infectious conditions, we wished to identify factors that led to CD127 expression in chronic inflammatory diseases and pursued TNF as a plausible candidate as its levels correlated with CD127 expression (Fig. 1i). TNF treatment consistently upregulated CD127 in monocytes, albeit to a lesser extent than LPS (Fig. 2f,g). Importantly, clinically applied TNF blockade treatment significantly reduced $I L 7 R$ expression in RA monocytes (Fig. 2h), further solidifying a role for TNF in upregulation of CD127 in vivo. Next, we wished to investigate whether CD127 in activated monocytes was functional given that another subunit of IL-7 receptor, the $I L 2 R G$-encoding common gamma chain, was constitutively expressed in human monocytes (Extended Data Fig. 3d). IL-7 treatment robustly induced STAT5 tyrosine phosphorylation in LPS-activated monocytes but not in resting monocytes (Fig. 2i) with T cells serving as positive controls (Extended Data Fig. 3e), indicating that activated human monocytes were competent for IL7 receptor signaling.

Given the heterogeneity of CD127 expression, we reasoned that activated human monocytes may display functional diversity and subjected LPS-activated monocytes to single cell expression profiling. Unsupervised hierarchical clustering revealed four groups of cells with differential gene expression patterns (Fig. 3a,b). Interestingly, IL7R exhibited a gradient pattern among four clusters, with the highest expression in cluster 1 and the lowest in cluster 4 (Fig. 3c). To quantitatively assess the inflammatory phenotypes of these cells, we devised a numeric index 'inflammatory score' based on an algorithm ${ }^{14}$ reflecting expression levels of 8 representative prototypical inflammatory genes (see 'Methods' for details). Inflammatory score inversely correlated with the expression of $I L 7 R$, with the highest level shown for cluster 4 that exhibited the 
lowest level of $I L 7 R$ (Fig. 3d), a trend that could also be clearly visualized for individual inflammatory genes (Fig. 3e). To validate the differences of inflammatory responses observed from single cell analyses, we sorted $\mathrm{CD} 127^{\text {high }}$ and $\mathrm{CD} 127^{\text {low }}$ LPS-activated monocytes (Fig. 3f). Consistent with the single cell results, CD127(IL7R $)^{\text {low }}$ monocytes produced significantly higher levels of inflammatory mediators such as IL-6 and TNF (Fig. 3g,h), demonstrating that within a highly defined system consisting of purified monocytes and a single stimulus, human monocytes displayed the remarkably diverse range of inflammatory responses.

Having observed the inverse correlation between CD127 and inflammatory responses, we wished to examine whether CD127 was causally related to inflammation by knocking down $I L 7 R$ in monocytes with RNA interference. Downregulation of IL7R resulted in markedly upregulated expression of IL6 and TNF (Fig. 3i), implicating a negative role for monocytic CD127 in inflammation. To characterize the chromatin accessibility landscape of CD127 $7^{\text {high }}$ and CD127 $7^{\text {low }}$ monocytes, we subjected two populations to ATAC-seq (Extended Data Fig. 4a) and identified a highly specified fraction of open chromatin regions in CD127 high monocytes (Fig. 3j,k) that displayed enhancer-like features marked by H3K27ac and H3K3me1 modifications ${ }^{15}$ (Extended Data Fig. 4b,c) and enriched in binding motifs for several transcription factors with STAT5 being the most prominent (Fig. 31). Pharmacological inhibition of STAT5 led to upregulation of $I L 6$ and $T N F$ expression (Fig. 3m), in line with the effects of $I L 7 R$ abrogation. To elucidate the mechanisms underlying CD127-STAT5-mediated effects, CD127 ${ }^{\text {high }}$ and CD127 $7^{\text {low }}$ population were profiled by RNA-seq (Extended Data Fig. 5a,b), revealing that transcription factor c-Maf, encoded by the $M A F$ gene, was relatively highly expressed in CD127 $7^{\text {high }}$ cells in a STAT5-dependent manner (Extended Data Fig. 5c,d). Knocking down $M A F$ expression resulted in upregulation of IL6 and TNF (Extended Data Fig. 5e,f). Of note, $M A F$ is a direct STAT5 target gene as shown by occupancy of STAT5 at a consensus binding site upstream of the $M A F$ transcription start site (Extended Data Fig. 5g). Together, the above results implicated that the CD127STAT5-c-Maf axis exerted anti-inflammatory effects, contributing to the functional heterogeneity in human inflammatory monocytes.

Upon characterizing the hypo-inflammatory phenotypes of CD127 high cells in vitro, we next wished to validate these findings in vivo in human disease settings. In BALF monocytes/macrophages from COVID19 patients, $I L 7 R^{+}$population did not extensively overlap with the highly inflammatory cells and exhibited minimal inflammatory properties (Fig. 4a,b). Consistent with the in vitro observations, $I L 7 R^{+}$cells expressed heightened levels of $M A F$ relative to the highly inflammatory monocytes (Extended Data Fig. 6a). Importantly, in mild COVID-19 patients, $I L 7 R^{+}$cells constituted $48 \%$ of all BALF monocytes/macrophages yet in stark contrast, such percentage was merely $17 \%$ in severe COVID-19 patients (Fig. 4c). The differential $I L 7 R$ patterns in monocytes were not due to the global differences in expression levels as lymphocytic $I L 7 R$ did not significantly differ between mild and severe patients (Extended Data Fig. 6b). These results indicated that CD127 indeed marked a population of hypo-inflammatory monocytes/macrophages in vivo and suggested that the prevalence of $I L 7 R^{+}$monocytes/macrophages likely correlated with subdued inflammation 
and favorable disease outcomes. In addition to COVID-19, $I L 7 R^{+}$population in RA synovial monocytes and peripheral blood monocytes were also largely non-overlapping with the highly inflammatory counterparts and displayed minimal inflammatory features (Fig. 4d,e and Extended Data Fig. 6c,d).

To extrapolate common features of $I L 7 R^{+}$monocytes/macrophages from various disease conditions, a recently developed bioinformatics method specializing in incorporating single cell sequencing data sets from multiple sources ${ }^{16}$ was used to run integrated analyses of three data sets: COVID-19 BALF monocytes/macrophages, RA synovial monocytes and in vitro LPS-activated monocytes. Unsupervised clustering identified 10 cellular subsets, with the cluster 10 classified as tissue resident alveolar macrophages from the COVID-19 samples (Extended Data Fig. 6e-g) and thus being excluded from the subsequent analyses intending to discover common monocyte characteristics regardless of tissue origins. The remaining 9 clusters (1-9) represented integrated monocyte subsets present in all three tissue sources (Fig. 4f), with cluster 2 prominently featured by high $I L 7 R$ expression (Fig. 4g,h) and sharing high degree of similarity among three conditions (Fig. 4i). Common signature genes of cluster 2 revealed a profile that was distinct from any of the currently known monocyte/macrophage subsets, with $I L 7 R$ unambiguously identified as the top marker gene (Fig. 4j). Of note, cluster 2 phenotypically differed from the 'M2-like' cells defined by markers such as CD163 (Extended Data Fig. 6h).

In summary, we identified a subset of monocytes marked by CD127 in human infectious and inflammatory diseases but not in mice, and named this subset M127 (Extended Data Fig. 7). We further characterized the inflammatory signals that induced M127 and recapitulated M127 phenotypes with an in vitro system. CD127 did not only serve as a surface marker for this population but also actively transmitted local IL-7 cues $^{17}$ to promote a STAT5-coordiated anti-inflammatory program, resulting in hypoinflammatory phenotypes amid the overall inflammatory tissue environments. As of the knowledge of the current study, M127 from multiple disease conditions and multiple tissues such as COVID-19 lungs and RA joints shared common functional features and gene signatures, albeit it would be interesting and desirable to assess whether the depicted M127 phenotypes could be observed in additional human disease settings. Given the unique presence of this population in human inflammatory diseases, especially the correlation of M127 expansion with favorable disease outcomes in COVID-19, it is highly conceivable to propose M127 as a potential therapeutic target for inflammatory disorders.

\section{Methods}

Cell culture and reagents

PBMCs of anonymous healthy donors were isolated from buffy coats purchased from the Beijing Red Cross Blood Center using density gradient cell separation by Ficoll (Lymphoprep ${ }^{\mathrm{TM}}$, STEMCELL Technologies) following the protocol approved by the Institutional Review Board of School of Medicine, Tsinghua University. The private information of anonymous blood donors was inaccessible to investigators. PBMCs 
191

192

193

194

195

196

197

198

199

200

201

202

203

204

205

206

207

208

209

210

211

212

213

of RA patients were obtained from Peking Union Medical College Hospital using the protocol that was approved by the Institutional Review Board of Peking Union Medical College Hospital. CD14 ${ }^{+}$Monocytes were further isolated from PBMCs using anti-CD14 magnetic beads (130-050-201, Miltenyi Biotec). CD14 ${ }^{+}$ monocytes were cultured in RPMI 1640 medium (10040CM, Corning) supplemented with 10\% (vol/vol) fetal bovine serum (FBS) (Gibco) and human recombinant M-CSF (300-25, Peprotech) (10 ng/ml). LPS (Escherichia coli O127:B8, Sigma-Aldrich), human recombinant IL-7 (200-07, Peprotech), human recombinant TNF (H8916, Sigma-Aldrich) or chemical inhibitors (SB203580 from Selleck, STAT5 Inhibitor from Santa Cruz and Bay 11-7082 from Sigma-Aldrich) were used as indicated for various experiments.

\section{Collection of lung tissues and immunohistochemistry}

Two cases of uninfected lung tissues and 3 cases of COVID-19 lung tissues were from Biobank of Southwest Hospital, Third Military Medical University (Army Medical University). COVID-19 lung tissues were obtained during autopsy of the patients succumbing to SARS-CoV-2 infection. Pathologically normal lung tissues from pulmonary bulla patients were used as uninfected controls. Tissue collection and the following histological analyses were approved by the ethics committee of Southwest Hospital, Third Military Medical University (Army Medical University), and were in accordance with regulations issued by the National Health Commission of China and the Helsinki Declaration. Lung tissue sections were stained with hematoxylin for assessment of pulmonary architecture, and anti-CD68 (ab201340, abcam) and anti-CD127 (PA5-97870, Invitrogen) antibodies were used for immunohistochemistry. Specifically, lung sections were deparaffinized and rehydrated. Antigen retrieval was performed with the Improved Citrate Antigen Retrieval Solution (Beyotime) and incubated with $\mathrm{H}_{2} \mathrm{O}_{2}$ in dark for 15 min to block endogenous peroxidase activity. Slides were blocked with 10\% goat serum in TBS for 30 min at room temperature and stained with primary antibodies overnight at $4{ }^{\circ} \mathrm{C}$. Slides were washed three times with $0.1 \%$ TBS-Tween before incubation with HRP-conjugated secondary antibodies. Stained slides were washed again in PBS and stained with DAB (TIANGEN) in conjunction with a hematoxylin counterstain (Solarbio). After dehydration, sections were mounted in neutral balsam.

\section{Immunofluorescence histology}

COVID-19 lung tissues were collected as described above, and were washed and fixed overnight at $4^{\circ} \mathrm{C}$ in a solution of $1 \%$ paraformadehyde in PBS. The tissues were incubated in a solution of $30 \%$ sucrose in PBS and the mixture of $30 \%$ sucrose and OCT compound 4583 (Sakura Finetek) separately at $4^{\circ} \mathrm{C}$ overnight. The samples were then embedded in OCT, frozen in a bath of ethanol cooled with liquid nitrogen and stocked at $-80^{\circ} \mathrm{C}$. Frozen samples were cut at $10-\mu \mathrm{m}$ thickness and collected onto slides. Slides were dried at $50^{\circ} \mathrm{C}$ for $30 \mathrm{~min}$ and fixed in 1\% paraformaldehyde for $10 \mathrm{~min}$ and processed for staining. The tissues were permeabilized in $\mathrm{PBS} / 0.3 \%$ Triton $\mathrm{X}-100 / 0.3 \mathrm{M}$ glycine at $37^{\circ} \mathrm{C}$ for $30 \mathrm{~min}$ and blocked in $\mathrm{PBS} / 5 \%$ goat serum at room temperature for $1 \mathrm{~h}$. The tissues were then incubated with indicated primary antibodies diluted (anti-CD68, 1:100; anti-CD127, 1:500) in PBS/5\% goat serum at $4^{\circ} \mathrm{C}$ overnight, and washed in $\mathrm{PBS} / 0.2 \%$ 
Tween-20 at room temperature for 30 min three times. The tissues were incubated with Alexa dye-conjugated secondary antibodies (Alexa Fluor ${ }^{\mathrm{TM}} 488$ goat anti-mouse IgG, 1:500, B40941, Life Technologies; Alexa Fluor $^{\text {TM }} 555$ goat anti-rabbit IgG, 1:500, A27039, Invitrogen) and DAPI (1:200, C0060-1, Solarbio) in $\mathrm{PBS} / 0.5 \% \mathrm{BSA}$ at room temperature for $2 \mathrm{~h}$ and washed in PBS/0.2\% Tween-20 at room temperature for 1 $\mathrm{h}$ five times before mounting with SlowFade Diamond Antifade Mountant (S36963, Life Technologies).

Bronchoalveolar lavage fluid (BALF) collection for single cell RNA sequencing (scRNA-seq) Nine COVID-19 patients were enrolled from the Shenzhen Third People's Hospital. BALF collection from COVID-19 patients and healthy donors and following studies were conducted according to the principles expressed in the Declaration of Helsinki. Ethical approval was obtained from the Research Ethics Committee of Shenzhen Third People's Hospital (2020-112). Diagnosis of COVID-19 was based on clinical symptoms, exposure history, chest radiography and SARS-CoV-2 RNA positivity. Disease severity was defined as moderate, severe and critical, according to the 'Diagnosis and Treatment Protocol of COVID-19' by the National Health Commission of China. Approximately $20 \mathrm{ml}$ of BALF was obtained for each patient. BALF was directly processed within $2 \mathrm{~h}$ and all operations were performed in a BSL-3 laboratory. BALF cells were collected, counted, re-suspended, and subsequently processed for scRNA-seq library construction as described in our previous study ${ }^{18}$. According to the clinical diagnosis, nine enrolled patients included three moderate cases, one severe case and five critical cases. For the subsequent analyses of scRNA-seq data, given that there was only one clinically defined severe case, the patients were stratified into mild $(n=3)$ and severe $(\mathrm{n}=6$ including both severe and critical cases) groups.

\section{Mice}

The laboratory animal facility at Tsinghua University has been accredited by AAALAC (Association for Assessment and Accreditation of Laboratory Animal Care International), and the IACUC (Institutional Animal Care and Use Committee) of Tsinghua University approved the protocol used in this study for blood collection from mouse cheeks. C57BL/6J mice were bred and housed in isolated ventilated cages (maxima six mice per cage) at the specific pathogen free facility at Tsinghua University. The mice were maintained on a $12 / 12-\mathrm{h}$ light/dark cycle, $22-26^{\circ} \mathrm{C}, 40-70 \%$ humidity with sterile pellet food and water ad libitum.

\section{RNA extraction and quantitative PCR.}

Total RNA was extracted from cells using TRIzol ${ }^{\circledR}$ Reagent according to the manufacturer's procedure, and total RNA was reverse-transcribed to cDNA with Moloney Murine Leukemia Virus Reverse Transcriptase (2641B, TAKARA). Real-time quantitative PCR (qPCR) was performed in duplicates with SYBR Green Master Mix (A25742, Applied Biosystems) on StepOnePlus thermal cycler (Applied Biosystems). Primer sequences are listed in the Extended Data Table 1.

\section{Flow cytometry}


Upon indicated treatment, cells were collected and washed with staining buffer (PBS with 0.5\% BSA and 2 mM EDTA). Then, the surface markers were stained with the indicated fluorochrome-conjugated antibodies in 1:400 dilution for $30 \mathrm{~min}$ on ice in the dark. After staining, cells were washed three times with staining buffer and re-suspended in PBS for analysis in BD FACSFortessa or for fluorescence-activated cell sorting (FACS) in BD FACSAria III. Further data analysis was implemented using Flowjo software (Tree star). For intracellular staining, cells were treated with Golgistop (554724, BD Biosciences) for 4-5 h before collection. The routine stainings for surface markers were performed, after which the cells were fixed with $100 \mu \mathrm{l} /$ tube Fixation Buffer (420801, Biolegend) for 25 minutes at room temperature, and the fixed cells were permeabilized and stained in $1 \times$ Permeabilization Wash Buffer with fluorochrome-conjugated antibodies for 30 minutes on ice in the dark. The fixed and intracellularly stained cells were washed twice with $1 \times$ Permeabilization Wash Buffer and suspended in PBS for analysis. The fluorochrome-conjugated antibodies for targets of interest and fluorochrome-conjugated isotype control antibodies are listed in Extended Data Table 2.

\section{Immunoblotting}

Whole-cell lysates were prepared by direct lysis in sodium dodecyl sulfate (SDS) loading buffer. All samples for immunoblotting were denatured at $95^{\circ} \mathrm{C}$ for $10 \mathrm{~min}$. For immunoblotting analysis, denatured cell lysates were separated by $10 \%$ SDS polyacrylamide gel electrophoresis and transferred to a polyvinylidene fluoride membrane (Millipore) for probing with specific primary antibodies and HRP-conjugated secondary antibodies. SuperSignal ${ }^{\mathrm{TM}}$ West Pico Chemiluminescent Substrate (34580, Thermo Fisher Scientific) was used for detection. Relative density of blotting bands was quantified using Image J (v1.52a). Antibodies used for probing proteins of interest are listed in Extended Data Table 2.

\section{RNA interference.}

Immediately after isolation, primary human monocytes were nucleofected with On-Target plus SMARTpool siRNA purchased from Dharmacon Inc. specific for $I L 7 R, M A F$ or $M A P 3 K 3$. Non-targeting siRNA from GenePharma was used as control. Human Monocyte Nucleofector buffer (V4XP-3024, Lonza) and the Lonza 4D-Nucleofector ${ }^{\mathrm{TM}}$ platform were used according to the manufacturers' instructions with human monocytes nucleofection program. The nucleofected monocytes were cultured in RPMI 1640 medium (Corning) supplemented with 10\% (vol/vol) FBS (Gibco) and human recombinant M-CSF (Peprotech) (20 ng/ml) for $48 \mathrm{~h}$ before the following experiments.

\section{Chromatin immunoprecipitation (ChIP) assay}

For STAT5 ChIP assays, THP-1 cells were stimulated with LPS $(100 \mathrm{ng} / \mathrm{ml})$ for $6 \mathrm{~h}$ and subsequently with IL-7 $(10 \mathrm{ng} / \mathrm{ml})$ for $30 \mathrm{~min}$. 10-20 $\times 10^{6}$ cells per condition were fixed in 1\% methanol-free formaldehyde (Thermo Scientific) for $8 \mathrm{~min}$ at room temperature followed by quenching with $125 \mathrm{mM}$ glycine for another 5 min. ChIP assay was performed using the SimpleChIP enzymatic ChIP kit (Cell Signaling Technology) 
according to the manufacturer's instructions. The DNA-protein complexes were immunoprecipitated using $5.0 \mu \mathrm{l}$ per sample of STAT5 antibody (9363S, Cell Signaling Technology), and IgG (2729P, Cell Signaling Technology) control was performed in an equally allocated DNA-protein complexes fraction as STAT5 ChIP samples. The immunoprecipitated DNA fragments were extracted with QIAquick PCR purification kit (QIAGEN) and subjected to qPCR assay for enrichment detection in MAF transcription start site (TSS) upstream GAS motif with primer pair, forward-AAGTGCAGTGCTATAAAGTTGTTT and reverseATGTTCAAGACGCTGGCTTA.

\section{RNA-seq}

Human $\mathrm{CD}_{1} 4^{+}$monocytes were stimulated with LPS $10 \mathrm{ng} / \mathrm{ml}$ for $6 \mathrm{~h}$, and CD127 high and CD127 populations for each donor were sorted by FACS. Total RNA was extracted from CD127 high and CD127 low cells using TRIzol ${ }^{\circledR}$ Reagent (Thermo Fisher Scientific) according to the manufacturer's procedure, and RNA samples were processed for library construction with TruSeq mRNA-seq Sample Preparation Kit (Illunima) and sequencing in BGI Genomics Co., Ltd. on a BGISEQ-500RS platform. Three independent sets of paired samples collected from three healthy donors were subjected to RNA-seq and the subsequent bioinformatics analyses.

Single cell RNA sequencing for RA PBMCs and LPS-activated monocytes

After the isolation or treatment, cells were frozen in FBS + 10\% DMSO for preservation in liquid nitrogen. The frozen cells were processed for scRNA-seq in BGI Genomics Co., Ltd. Single-cell capturing and downstream library constructions were performed using Chromium Single Cell 3' Reagent kits (10x Genomics) according to the manufacturer's protocol. The constructed libraries were sequenced on a BGI MGISEQ2000 platform.

Assay for transposase-accessible chromatin coupled with high-throughput sequencing (ATAC-seq) Human $\mathrm{CD}_{14}{ }^{+}$monocytes from healthy donors were stimulated with $10 \mathrm{ng} / \mathrm{ml}$ of LPS for $6 \mathrm{~h}$, and CD127 high and $\mathrm{CD} 127^{\text {low }}$ populations for each donor were sorted by FACS. Cells were pelleted by centrifugation for 10 min at $500 \mathrm{~g} 4^{\circ} \mathrm{C}$ using a swing rotor with low acceleration and brake settings. Cell pellets were washed once with $1 \times$ PBS and cells were pelleted again by centrifugation using the previous settings. Cell pellets were re-suspended in $50 \mu \mathrm{l}$ of lysis buffer (10 mM Tris- $\mathrm{HCl} \mathrm{pH} 7.4,10 \mathrm{mM} \mathrm{NaCl}, 3.0 \mathrm{mM} \mathrm{MgCl}, 0.5 \% \mathrm{NP}-40)$ and nuclei were pelleted by centrifugation for $30 \mathrm{~min}$ at $500 \mathrm{~g}, 4^{\circ} \mathrm{C}$ using a swing rotor with low acceleration and brake settings. Supernatant was discarded and nuclei were re-suspended in $50 \mu 1$ reaction buffer containing $5.0 \mu \mathrm{l}$ Tn5 transposase and $10 \mu \mathrm{l}$ of $5 \times$ TTBL buffer (TruePrepTM DNA Library Prep Kit V2 for Illumina, Vazyme Biotech). The reaction was incubated at $37^{\circ} \mathrm{C}$ for $30 \mathrm{~min}$. After the tagmentation, the transposed DNA fragments were purified by $1 \times$ AMPure XP beads (Beckman Coulter). PCR was performed to amplify the libraries for 9 cycles using the following PCR conditions: $72^{\circ} \mathrm{C}$ for $3 \mathrm{~min}$; $98^{\circ} \mathrm{C}$ for $30 \mathrm{~s}$; and thermocycling at $98^{\circ} \mathrm{C}$ for $15 \mathrm{~s}, 60^{\circ} \mathrm{C}$ for $30 \mathrm{~s}$ and $72^{\circ} \mathrm{C}$ for $3 \mathrm{~min}$; following by $72^{\circ} \mathrm{C} 5 \mathrm{~min}$. After the PCR 
reaction, libraries were purified with the $0.5 \times$ and $1.2 \times$ AMPure XP beads. DNA concentrations were measured with StepOnePlusTM Real-Time PCR System (Life Technologies) and library sizes were determined using Agilent 2100 Bioanalyzer. Libraries were sequenced on an Illumina Hiseq X-ten platform for an average of 20 million unique reads per sample. Three independent sets of paired samples collected from three healthy donors were subjected to ATAC-seq and the subsequent bioinformatics analyses.

\section{Next-generation sequencing (NGS) data alignment}

ATAC-seq pair-end reads were collected. Adapter sequences were trimmed from the ends of reads by Cutadapt (v1.14), and the reads that failed to pass the quality control $(\mathrm{Q}>10)$ were discarded. H3K27ac and H3K4me1 ChIP-seq data sets were downloaded from NCBI GEO DataSet under the GEO accessions: GSE85245 ${ }^{19}$. SRA files were converted to fastq files using fastq-dump included in SRA toolkit. Pair-end ATAC-seq reads were aligned to human genome (UCSC hg38) using Bowtie2 (v2.2.5) ${ }^{20}$ to generate alignment files of uniquely mapped pair-end fragments with maximum length in $1000 \mathrm{bp}$ and no more than one mismatch for each alignment seed with $15 \mathrm{bp}$ in length. ChIP-seq reads in fastq files were aligned to human genome (UCSC hg38) using Bowtie (v1.1.2) ${ }^{21}$ to generate alignment files of uniquely mapped reads with maximum allowed mismatch of $2(-\mathrm{m} 1-\mathrm{n} 2)$ for each alignment seed. ChIP-seq reads aligned to genome were extended to $150 \mathrm{bp}$ from their 3' end for further analysis. RNA-seq data were collected and single-end reads were aligned to human genome hg38 using TopHat (v2.1.0 $)^{22}$ with the parameters --min-segment-intron 50 --no-novel-indels --no-coverage-search, and only uniquely mapped reads were preserved.

\section{Identification of opened chromatin regions (OCR) by ATAC-seq}

The ATAC-seq alignment files for CD12 $7^{\text {high }}$ and CD127 $7^{\text {low }}$ monocytes from three donors were used to call peaks for significantly opened chromatin regions using MACS2 (v2.1.1) (FDR < 0.05). The peaks from six samples were merged as total OCRs in LPS treated monocytes. To identify the differentially opened chromatin regions between $\mathrm{CD} 127^{\text {high }}$ and $\mathrm{CD} 127^{\text {low }}$ monocytes, the ATAC-seq fragments were counted in each OCR for each sample by FeatureCounts (v1.5.0) ${ }^{23}$. Subsequently, the fragments count was normalized to count per million mapped fragment for each sample. The normalized fragments count was used to identify differentially opened chromatin regions by edgeR (v3.28.1 ${ }^{24}$. Mean values of (fragments count +1$)$ fold change (CD127 $7^{\text {high }} / \mathrm{CD} 127^{\text {low }}$ ) among three donors were $\log 2$ transformed, and differentially opened chromatin regions were identified by $\log 2$ transformed fold changes $\left(\mathrm{CD} 127^{\text {high }} / \mathrm{CD} 127^{\text {low }}\right) \geq 1$ or $\leq-1$ for $\mathrm{CD} 127^{\text {high }}$ monocytes feature OCRs or CD127 $7^{\text {low }}$ monocytes feature OCRs with cutoff of p-value $<0.05$. To visualize ATAC-seq and ChIP-seq signals around open chromatin regions of interest, we firstly counted ATAC-seq fragments (-fragLength given) and ChIP-seq extended reads (-fragLength 150) every $10 \mathrm{bp}$ from the center of OCR to $\pm 2.5 \mathrm{~kb}$ regions for each OCR by using annotatePeaks.pl program in HOMER $(\mathrm{v} 4.7 .2)^{25}$. The output counting matrices were used to generate average signals around OCRs by calculating the average fragments/reads count per bin (10 bp) per OCR. 
RNA-seq data analyses

For coverage of mapped RNA-seq reads in transcripts, the expression level of each gene transcript was calculated as normalized reads count per kilobase of transcript per million mapped reads (FPKM) using Cufflinks (v2.2.1) ${ }^{26}$. Differential gene expression between CD127 $7^{\text {high }}$ and CD127 $7^{\text {lwo }}$ monocytes from three donors was identified using DESeq2 (v1.27.9) ${ }^{27}$. Genes with p-value $<0.05$ and mean $(\mathrm{FPKM}+1)$ fold changes $\left(\mathrm{CD} 127^{\text {high}} / \mathrm{CD} 127^{\text {low }}\right) \geq 1.5$ or $\leq 0.67$ among three donors were defined as highly expressed genes in $\mathrm{CD} 127^{\text {high }}$ monocytes or highly expressed genes in CD127 $7^{\text {low }}$ monocytes, respectively.

scRNA-seq data analyses

For scRNA-seq of COVID-19 patients' BALF cells, the Cell Ranger Software Suite (v.3.1.0) was used to perform sample de-multiplexing, barcode processing and single-cell 5' unique molecular identifier (UMI) counting. Specifically, splicing-aware aligner STAR was used in FASTQs alignment. Cell barcodes were then determined based on the distribution of UMI counts automatically, and the gene-barcode matrices were saved for downstream analysis. In addition, one additional healthy control was acquired from the GEO database under accession number GSE128033. All samples were loaded as Seurat objects by using Seurat $(\mathrm{v} 3.2 .1)^{14}$, quality control for each cell were done with criteria as following: gene number between 200 and 6,000 , UMI count $>1,000$ and mitochondrial gene percentage $<0.1$. All samples were further integrated to remove the batch effects with the parameter settings of the first 50 dimensions of canonical correlation analysis (CCA) and principal-component analysis (PCA). Integrated Seurat project was first normalized, and top 2,000 variable genes were then identified by using the Seurat analysis pipeline that has been described in our previous studies. Gene expression scaling and PCA was performed using the top 2,000 variable genes. Then UMAP was performed on the top 50 principal components for visualization, and graph-based clustering was simultaneously performed on the PCA-reduced data with the 1.2 resolution setting. According to the clustering result, 32 clusters were identified, and the annotations for each cluster were implemented based on the expression of marker genes that were used in our previous study ${ }^{18}$. Monocytes/macrophages $\left(\mathrm{CD} 14^{\text {high }}\right.$ CD68 ${ }^{\text {high }}$ ) were extracted from the total BALF cells after annotation, and re-clustering (PCA and UMAP) was performed. The clusters showing expression of both monocyte/macrophage marker genes and $\mathrm{T}$ cell marker genes were excluded as doublets. Only the cells from COVID-19 patients were used for downstream analyses on both total BALF cells and monocytes/macrophages.

RA synovial scRNA-seq data sets ${ }^{13}$ were downloaded from ImmPort with the study accession code of SDY998. The reduction and clustering result from the original study were used. The monocytes clusters were extracted for downstream analysis.

The Cell Ranger Software Suite (v.3.1.0) was used to perform sample de-multiplexing, barcode processing and single-cell 5' unique molecular identifier (UMI) counting, and gene-barcode matrices were generated for LPS-treated human CD14+ monocytes and RA patient's PBMCs. The gene-barcode matrices were loaded as Seurat objects, and quality control for each cell was performed with criteria for LPS-treated human $\mathrm{CD}_{14}^{+}$monocytes (gene number between 200 and 4,500, UMI count > 1,000 and mitochondrial gene 
413 percentage $<0.15$ ) and RA PBMCs (gene number between 200 and 6,000, UMI count $>1,000$ and 414 mitochondrial gene percentage $<0.1$ ). After quality control, top 2,000 variable genes were identified, and gene expression scaling, PCA and UMAP clustering were performed for each data set. Marker genes for each cluster in each scRNA-seq data set were identified by using FindAllMarkers function in Seurat. According to the expression of well-studied PBMC marker genes, each cluster of RA PBMCs was annotated with certain cell type, and monocyte clusters were extracted for downstream analysis.

$I L 7 R^{+}$cells were identified based on the normalized expression $(>0)$ of $I L 7 R$ for each cells in each scRNA-seq dataset. Inflammatory score was calculated based on the normalized average expression of eight inflammatory genes: TNF, IL6, IL8, CCL2, CCL3, CCL4, CCL8 and CXCL10, which is implemented by AddModuleScore function in Seurat with 100 control for each inflammatory gene.

Integration of COVID-19 monocytes/macrophages, RA synovial monocytes and LPS-treated monocytes was implemented by a recently developed SCALEX method based on the original SCALE method $^{16}$. Leiden clustering and UMAP visualization were performed based on the features extracted by SCALEX for integrated monocytes/macrophages, and marker genes for each cluster were identified. Correlation between clusters among three datasets were calculated as Pearson correlation coefficient, and the negative values were normalized to zero for heat map presentation.

Statistical analysis

Types of statistical tests are indicated in figure legends. Statistical analyses were performed using GraphPad Prism Software (GraphPad Software Inc., La Jolla, CA, USA) for Student's $t$ test, and Wilcoxon rank-sum test was implemented using R (v.4.0.2). A value of $P<0.05$ was considered statistically significant.

\section{References:}

4361 Merad, M. \& Martin, J. C. Pathological inflammation in patients with COVID-19: a key role for monocytes and macrophages. Nature reviews. Immunology 20, 355-362 (2020).

4382 Donlin, L. T. et al. Insights into rheumatic diseases from next-generation sequencing. Nat Rev $439 \quad$ Rheumatol 15, 327-339 (2019).

4403 Ermann, J., Rao, D. A., Teslovich, N. C., Brenner, M. B. \& Raychaudhuri, S. Immune cell profiling 441 to guide therapeutic decisions in rheumatic diseases. Nat Rev Rheumatol 11, 541-551 (2015).

4424 Shi, C. \& Pamer, E. G. Monocyte recruitment during infection and inflammation. Nature reviews. Immunology 11, 762-774 (2011).

4445 van der Poll, T., van de Veerdonk, F. L., Scicluna, B. P. \& Netea, M. G. The immunopathology of sepsis and potential therapeutic targets. Nature reviews. Immunology 17, 407-420 (2017).

4466 Mildner, A., Yona, S. \& Jung, S. A close encounter of the third kind: monocyte-derived cells. Adv Immunol 120, 69-103 (2013).

4487 Auffray, C., Sieweke, M. H. \& Geissmann, F. Blood monocytes: development, heterogeneity, and relationship with dendritic cells. Annu Rev Immunol 27, 669-692 (2009). 
4508 Guilliams, M., Mildner, A. \& Yona, S. Developmental and Functional Heterogeneity of Monocytes. 451 Immunity 49, 595-613 (2018).

4529 Nathan, C. \& Ding, A. Nonresolving inflammation. Cell 140, 871-882 (2010).

$45310 \quad$ Vabret, N. et al. Immunology of COVID-19: Current State of the Science. Immunity 52, 910-941 454 (2020).

45511 Barata, J. T., Durum, S. K. \& Seddon, B. Flip the coin: IL-7 and IL-7R in health and disease. Nat $456 \quad$ Immunol 20, 1584-1593 (2019).

45712 Kalliolias, G. D. \& Ivashkiv, L. B. TNF biology, pathogenic mechanisms and emerging therapeutic $458 \quad$ strategies. Nat Rev Rheumatol 12, 49-62 (2016).

45913 Zhang, F. et al. Defining inflammatory cell states in rheumatoid arthritis joint synovial tissues by 460 integrating single-cell transcriptomics and mass cytometry. Nat Immunol 20, 928-942 (2019). Stuart, T. et al. Comprehensive Integration of Single-Cell Data. Cell 177, 1888-1902 e1821 (2019). Smale, S. T. \& Natoli, G. Transcriptional control of inflammatory responses. Cold Spring Harb Perspect Biol 6, a016261 (2014).

Xiong, L. et al. SCALE method for single-cell ATAC-seq analysis via latent feature extraction. Nat Commun 10, 4576 (2019).

19 Novakovic, B. et al. beta-Glucan Reverses the Epigenetic State of LPS-Induced Immunological Tolerance. Cell 167, 1354-1368 e1314 (2016).

20 Langmead, B. \& Salzberg, S. L. Fast gapped-read alignment with Bowtie 2. Nat Methods 9, 357-359 (2012).

21 Langmead, B., Trapnell, C., Pop, M. \& Salzberg, S. L. Ultrafast and memory-efficient alignment of short DNA sequences to the human genome. Genome Biol 10, R25 (2009).

Kim, D. et al. TopHat2: accurate alignment of transcriptomes in the presence of insertions, deletions and gene fusions. Genome Biol 14, R36 (2013).

Liao, Y., Smyth, G. K. \& Shi, W. featureCounts: an efficient general purpose program for assigning sequence reads to genomic features. Bioinformatics 30, 923-930 (2014). expression analysis of digital gene expression data. Bioinformatics 26, 139-140 (2010).

Heinz, S. et al. Simple combinations of lineage-determining transcription factors prime cis-regulatory elements required for macrophage and B cell identities. Mol Cell 38, 576-589 (2010).

26 Trapnell, C. et al. Differential gene and transcript expression analysis of RNA-seq experiments with TopHat and Cufflinks. Nat Protoc 7, 562-578 (2012).

27 Love, M. I., Huber, W. \& Anders, S. Moderated estimation of fold change and dispersion for RNA- 
489 Acknowledgements: We thank the patients and their families for their contribution to scientific research. 490 We also thank clinical staffs and the COVID-19 Pathology Team from Third Military Medical University 491 and Shanghai Jiao Tong University for performing the autopsy work. This research was supported by 492 National Natural Science Foundation of China grants (31725010 and 31821003 to X.H), Emergency Project 493 from Chongqing Health Commission (2020NCPZX01 to X.W.B.), Tsinghua University COVID-19 494 Scientific Research Program (2020Z99CFZ024 to X.H.), and funds from Tsinghua-Peking Center for Life 495 Sciences (to X.H.).

496 Author contributions: Bin Zhang performed experiments and bioinformatic analyses, analyzed and 497 interpreted data, and wrote the manuscript. Yuan Zhang performed experiments and analyzed and interpreted 498 data. Lei Xiong and Yuzhe Li assisted with integration of single cell RNA-seq datasets. Yunliang Zhang 499 performed some of the monocyte experiments and assisted with single cell RNA-seq data analyses. Jiuliang 500 Zhao, Hui Jiang, and Can Li assisted with collection of rheumatoid arthritis patient samples. Yunqi Liu 501 performed STAT5 ChIP experiments. Xindong Liu, Haofei Liu, and Yi-Fang Ping performed pathological 502 analyses of COVID-19 patient tissue samples. Qiangfeng Cliff Zhang directed the integration of single cell 503 RNA-seq datasets and provided advices on bioinformatics. Zheng Zhang provided COVID-19 BALF single 504 cell RNA-seq data sets and valuable advices. Xiu-Wu Bian directed pathological analyses of COVID-19 505 patient tissue samples. Yan Zhao provided rheumatoid arthritis patient samples and advices on the related 506 experiments. Xiaoyu $\mathrm{Hu}$ conceptualized the project, supervised experiments, analyzed and interpreted data, 507 and wrote the manuscript.

508 Competing interests: We declare no competing conflicts of interest.

509 Materials \& Correspondence: Correspondence and requests for materials should be addressed to X.H.

510 Data availability: Sequencing data sets are deposited in the Genome Expression Omnibus with assigned 511 accession numbers as follows: RNA-seq and ATAC-seq in GSE159118, healthy donor scRNA-seq in 512 GSE159113, RA scRNA-seq in GSE159117 and COVID-19 BALF scRNA-seq in GSE145926. 

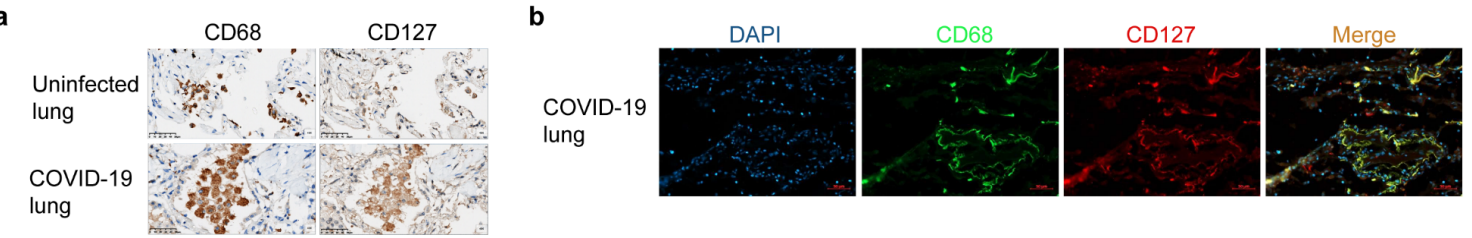

c

COVID-19 BALF mono/mac

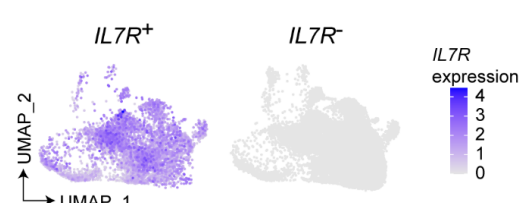

g PBMCs

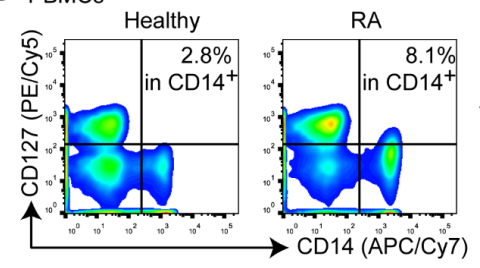

j

RA synovial monocytes

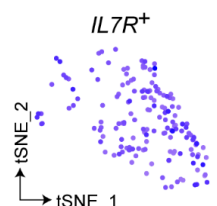

d

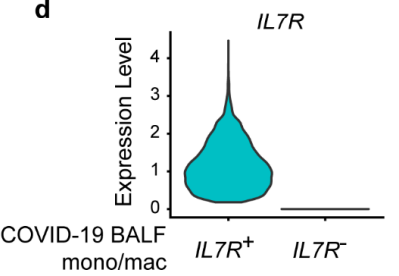

h

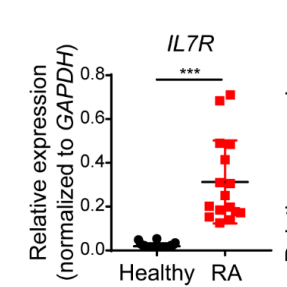

k
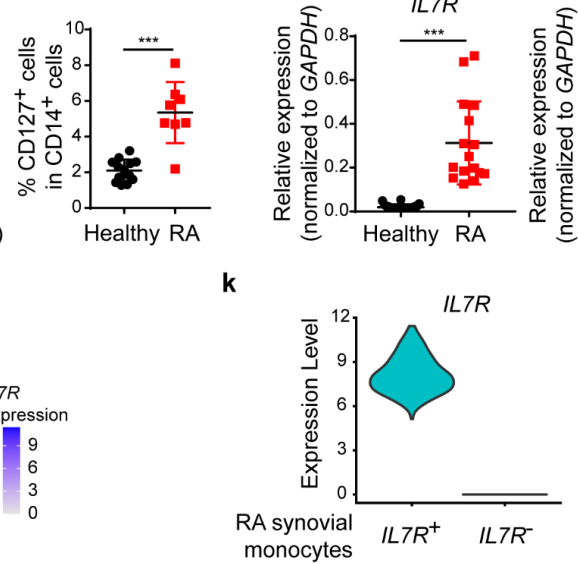

e

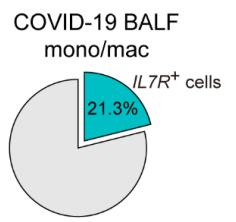

$\mathrm{mono} / \mathrm{mac}$
COLF

f

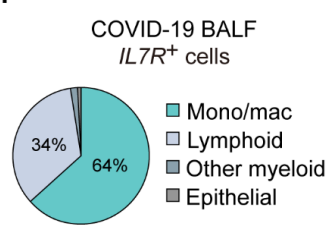

i

\section{Figure 1. CD127 ${ }^{\text {high }}$ monocytes/macrophages are present in human inflammatory conditions}

(a) Immunohistochemical analysis of CD68 and CD127 expression in lung tissues sections. Uninfected lung tissues and COVID-19 lung tissues were obtained during autopsy following the protocol described in Methods. One representative result from tissue sections of three COVID-19 cases is shown. Scale bars represent $50 \mu \mathrm{m}$. (b) Immunofluorescence staining for DAPI (blue), CD68 (green) and CD127 (red) in sections from COVID-19 lung tissues. One representative result from tissue sections of three COVID-19 cases is shown. Scale bars represent $50 \mu \mathrm{m}$. (c) UMAP projection of $I L 7 R^{+}$and $I L 7 R^{-}$ monocytes/macrophages (mono/mac) in broncho-alveolar lavage fluid (BALF) from COVID-19 patients. $I L 7 R$ expression among cells was shown by color as indicated. (d) Violin plot shows the expression of $I L 7 R$ in $I L 7 R^{+}$and $I L 7 R^{-}$BALF mono/mac from COVID-19 patients. (e) Pie graph shows the percentage of $I L 7 R^{+}$ cells in BALF mono/mac from COVID-19 patients. (f) Pie graph shows the percentages of each cell type in total $I L 7 R^{+}$BALF cells from COVID-19 patients. (g) PBMCs were isolated from the peripheral blood of healthy donors $(n=13)$ and rheumatoid arthritis $(\mathrm{RA})$ patients $(\mathrm{n}=8)$, and CD127 expression was measured by flow cytometry analysis (FACS). Representative FACS plot (left) and cumulative percentages (right) of $\mathrm{CD} 127^{+}$population are shown. $* * * P<0.001$ by unpaired Student's $t$ test. Error bars indicate means $\pm \mathrm{SD}$. (h, i) $\mathrm{CD}_{14}{ }^{+}$monocytes were isolated from PBMCs of healthy donors and RA patients, and mRNA of IL7R and $T N F$ was measured using quantitative PCR (qPCR) (h). Relative expression was normalized to internal control $(G A P D H)$. Linear regression analysis was used to compare the expression of $T N F$ and $I L 7 R$ in CD14 
532 monocytes from RA patients (i). Correlation coefficient (r) and p-value for coefficient are shown in the panel. $533 * * P<0.01, * * * P<0.001$ by unpaired Student's $t$ test. Error bars indicate means \pm SD. (IL7R, Healthy $\mathrm{n}=20$ 534 and RA $\mathrm{n}=16 ; T N F$, Healthy $\mathrm{n}=8$ and RA $\mathrm{n}=16)(\mathrm{j}) \mathrm{t}-\mathrm{SNE}$ projection of $I L 7 R^{+}$and $I L 7 R^{-}$cells in RA 535 synovial monocytes. $I L 7 R$ expression among cells was shown by the indicated color. (k) Violin plot shows 536 the expression of $I L 7 R$ in $I L 7 R^{+}$and $I L 7 R^{-}$RA synovial monocytes. (l) Pie graph shows the percentage of $537 I L 7 R^{+}$cells in RA synovial monocytes. 
a

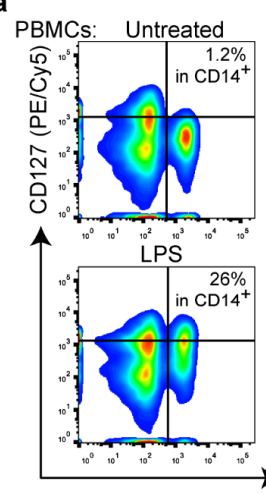

d

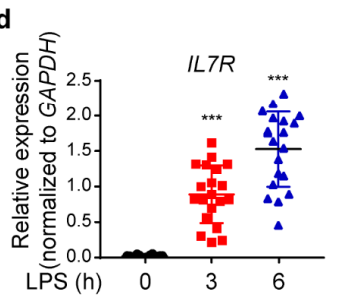

g

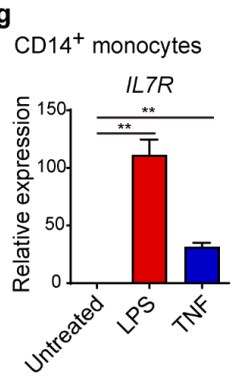

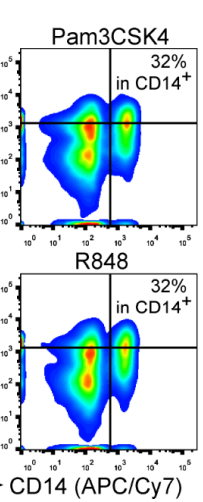

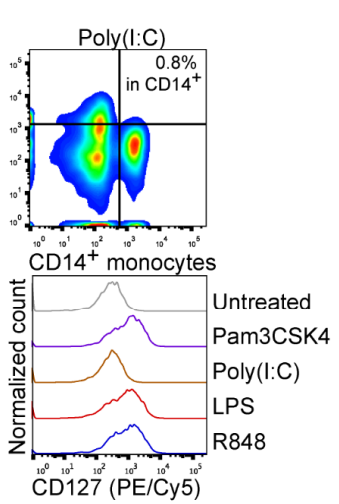

CD14 ${ }^{+}$monocytes

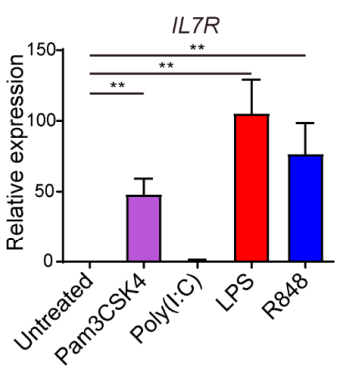

C

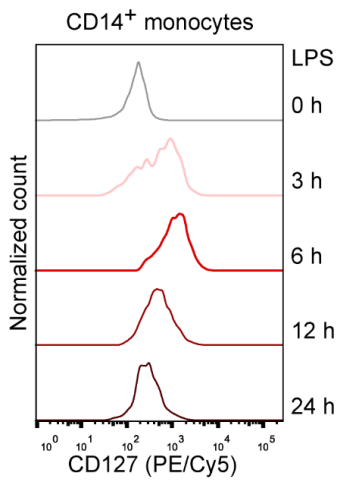

f

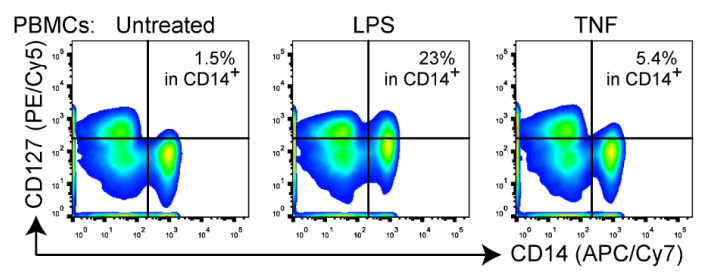

h RA CD $14^{+}$monocytes 듬 ${ }^{1.5} 1 \stackrel{\text { IL7R }}{* \text { * B B for }}$

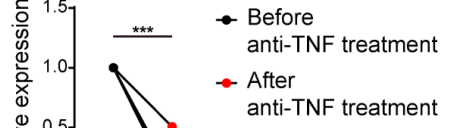

anti-TNF treatment

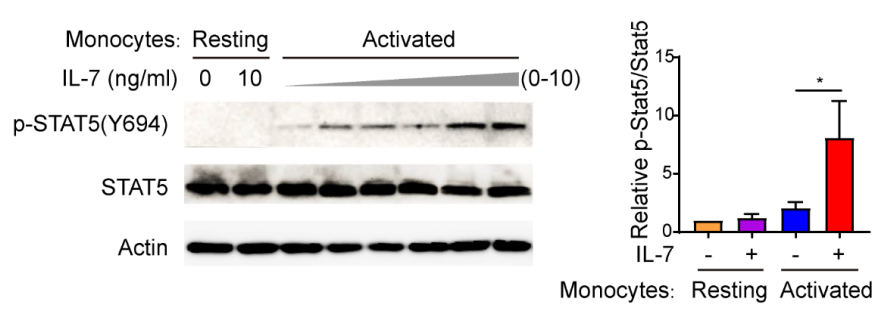

Figure 2. CD127 $7^{\text {high }}$ monocytes are inducible by inflammatory stimuli and competent for CD127STAT5 signaling

(a) PBMCs from healthy blood donors were stimulated with Pam3CSK4 (100 ng/ml), Poly(I:C) (1 $\mu \mathrm{g} / \mathrm{ml})$, LPS $(10 \mathrm{ng} / \mathrm{ml})$ or R848 $(1 \mu \mathrm{g} / \mathrm{ml})$ for $6 \mathrm{~h}$, and CD127 expression was measured by FACS. Histograms in bottom right shows the CD127 staining signal in $\mathrm{CD}_{1} 4^{+}$cells under each condition as indicated. One representative FACS result from three biological replicates is shown. (b) CD $14^{+}$monocytes from healthy donors' PBMCs were stimulated with Pam3CSK4 (100 ng/ml), Poly(I:C) (1 $\mu \mathrm{g} / \mathrm{ml})$, LPS (10 ng/ml) or R848 $(1 \mu \mathrm{g} / \mathrm{ml})$ for $3 \mathrm{~h}$. The mRNA of $I L 7 R$ was measured by qPCR. Relative expression was normalized to internal control $(G A P D H)$ and expressed relative to untreated sample. ${ }^{*} P<0.01$ by paired Student's $t$ test. Data are shown as means \pm SD of four independent experiments with one healthy donor for each data set. (c) CD14 monocytes from healthy donor's PBMCs were treated with $10 \mathrm{ng} / \mathrm{ml}$ LPS for various time points as indicated, and the expression of CD127 was measured by FACS. (d) CD14 ${ }^{+}$monocytes were treated with $10 \mathrm{ng} / \mathrm{ml}$ LPS for $3 \mathrm{~h}$ and $6 \mathrm{~h}$, the mRNA of $I L 7 R$ was measured by real time qPCR. Relative expression was normalized to $G A P D H$ as internal control. Each data point represented an independent experiment from one healthy donor. $* * * P<0.001$ by unpaired Student's $t$ test. Error bars indicate means \pm SD of 20 independent experiments. (e) Three different monocyte subsets were FACS-sorted from PBMCs as shown in Extended Data Fig. 2f. The 
556 sorted cells were treated with or without $10 \mathrm{ng} / \mathrm{ml}$ LPS for $3 \mathrm{~h}$. The expression of $I L 7 R$ was measured by q557 PCR. Relative expression was normalized to internal control (GAPDH) and expressed relative to LPS558 untreated $\mathrm{CD} 14^{+} \mathrm{CD} 16^{++}$sample. ${ }^{* *} P<0.01, * P<0.05$ by unpaired Student's $t$ test. Data are shown as means $559 \pm$ SD of three independent experiments with one healthy donor for each data set. (f, $\mathbf{g})$ PBMCs from healthy 560 donor were treated with LPS $(10 \mathrm{ng} / \mathrm{ml})$ or recombinant human TNF $(100 \mathrm{ng} / \mathrm{ml})$. Upon $6 \mathrm{~h}$ LPS or TNF 561 treatment, CD127 expression was measured by FACS. Representative FACS distribution is shown (f). 562 mRNA of $I L 7 R$ was measured in $3 \mathrm{~h}$ LPS or TNF treated monocytes by qPCR (g). Relative expression was 563 normalized to internal control $(G A P D H)$ and expressed relative to untreated sample. $* * P<0.01$ by paired 564 Student's $t$ test. Data are shown as means \pm SD of three independent experiments with one healthy donor for 565 each data set. (h) mRNA level of $I L 7 R$ was measured by real time qPCR in monocytes from RA patients (n $566=5$ ) before and after Etanercept anti-TNF treatment for 2 months. Relative expression was normalized to 567 internal control $(G A P D H)$. ${ }^{* * *} P<0.001$ by paired Student's $t$ test. (i) CD14 ${ }^{+}$monocytes were pre-treated with 568 or without LPS for $6 \mathrm{~h}$, then followed by various doses of recombinant human IL-7 (from $1 \mathrm{pg} / \mathrm{ml}$ to $10 \mathrm{ng} / \mathrm{ml}$ ) 569 for $30 \mathrm{~min}$. STAT5 activation was detected by western blotting. Actin was used as loading control. One 570 representative experiment out of three biological replicates is shown (left). The protein level of p571 STAT5(Y694) was quantified by densitometry, normalized to total STAT5 protein and expressed relative to 572 untreated (without LPS and IL-7) sample (right). $* P<0.05$ by paired Student's $t$ test. Data were expressed as 573 mean \pm SD of three independent experiments with one healthy donor for each data set. 
b

a Unsupervised clustering in LPS-treated monocytes

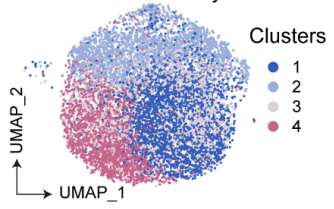

Cell clusters in LPS-treated
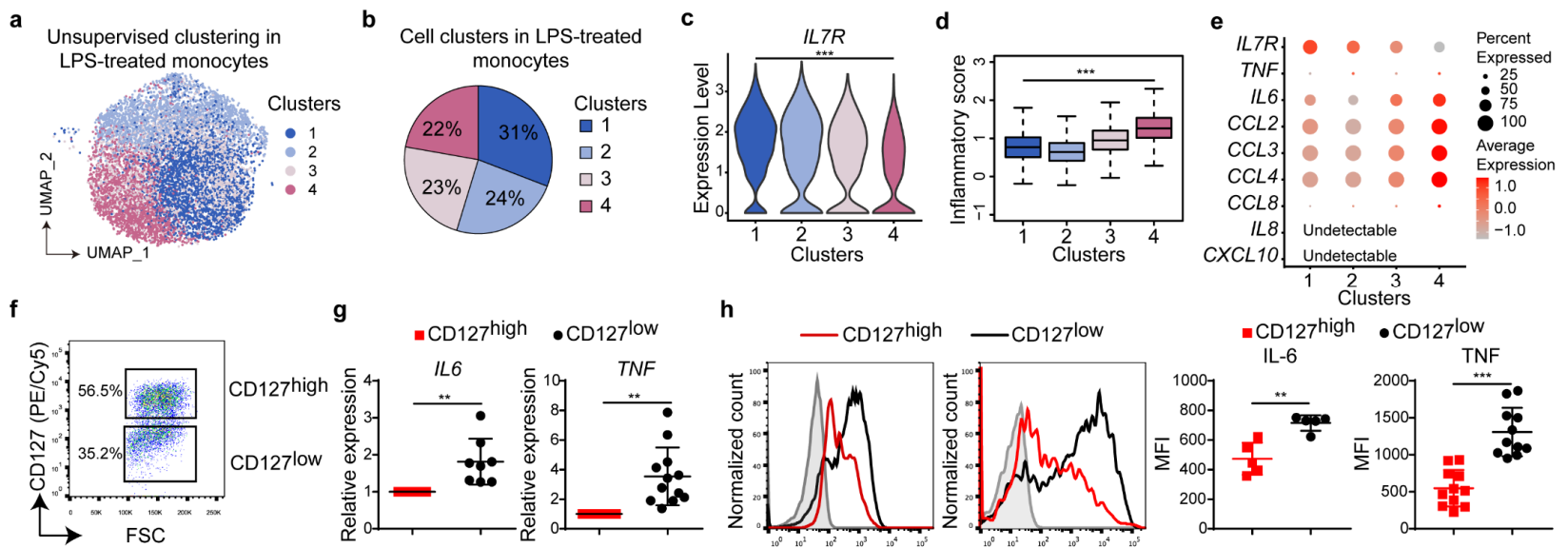

g $\quad$ CD127high $\bullet$ CD127low

$\mathbf{h}$
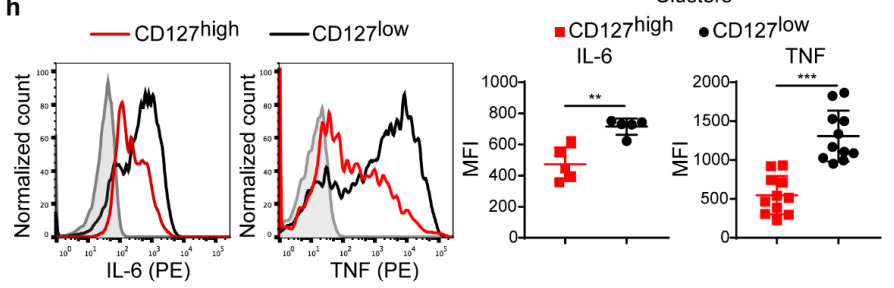

j
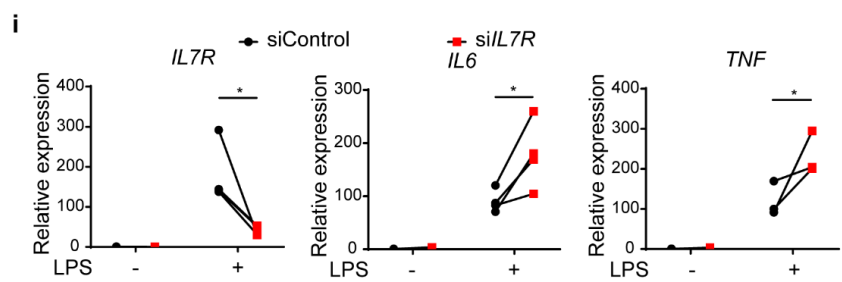

k

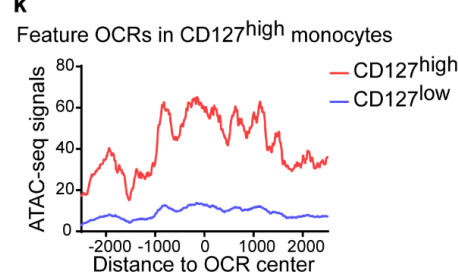

I

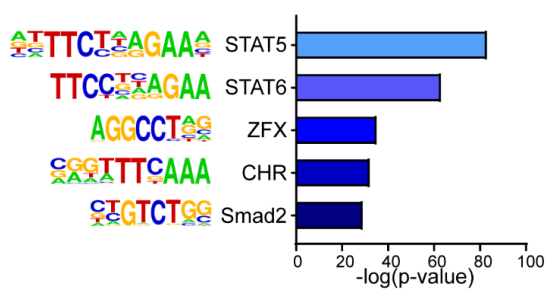

Open chromatin regions (OCR) in LPS-treated monocytes $2.9 \% \quad(n=11281)$

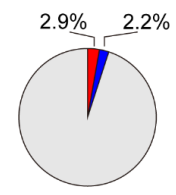

Feature OCRs in CD127 high monocytes

Feature OCRs in CD127 low monocytes

m
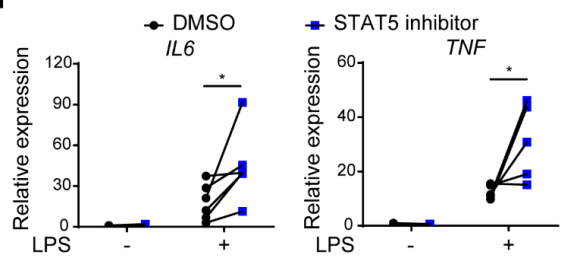

Figure 3. CD127 imposes heterogeneity in monocyte inflammatory responses mediated by CD127STAT5 axis

(a) UMAP projection of LPS $6 \mathrm{~h}$ treated human $\mathrm{CD} 14^{+}$monocytes that were subgrouped by unsupervised cluster analysis of scRNA-seq data. (b) Pie graph shows the percentages of each monocyte cluster shown in (A) in LPS-treated monocytes. (c) Violin plot shows the expression of $I L 7 R$ among four clusters of LPStreated monocytes. ${ }^{* * *} P<0.001$ by Wilcoxon rank-sum test. (d) Inflammatory score was defined by average expression of eight inflammatory genes, and box plot shows the inflammatory score distribution among four clusters of LPS-treated monocytes. ${ }^{* * *} P<0.001$ by Wilcoxon rank-sum test. (e) Heat map shows the expression of $I L 7 R$ and eight inflammatory genes for inflammatory score calculation among four clusters of LPS-treated monocytes. The scaled average expression levels for each gene and percentages of cells expressing each gene in each cluster were represented by color and size of the corresponding dots, respectively. (f, g) $\mathrm{CD} 127^{\text {high }}$ and $\mathrm{CD} 127^{\text {low }}$ populations were isolated from $6 \mathrm{~h} \mathrm{LPS-stimulated} \mathrm{CD} 14^{+}$ monocytes by FACS (f) and the mRNA levels of $I L 6$ and $T N F$ in two populations were measured by qPCR (g). Relative expression was normalized to internal control $(G A P D H)$ and shown relative to $\mathrm{CD} 127^{\text {high }}$ sample. $* * P<0.01,{ }^{*} * * P<0.001$ by paired Student's $t$ test. Data are shown as means \pm SD of eight independent experiments for IL 6 and twelve independent experiments for TNF. (h) Protein levels of IL-6 and TNF in $\mathrm{CD} 127^{\text {high }}$ and $\mathrm{CD} 127^{\text {low }}$ populations were measured by GolgiStop-utilized intracellular staining and analyzed by flow cytometry. One representative FACS plot (left) and cumulative mean fluorescence 
594 intensities (MFI) from five independent experiments for IL-6 and eleven independent experiments for TNF 595 (right) are shown. ${ }^{*} P<0.01, * * * P<0.001$ by paired Student's $t$ test. Data are shown as means \pm SD. (i) CD $14^{+}$ 596 monocytes were transfected with negative control or $I L 7 R$ specific short interfering RNA (siControl or $597 \mathrm{si} I L 7 R)$. Two days post transfection, cells were stimulated with LPS $(10 \mathrm{ng} / \mathrm{ml})$ for $3 \mathrm{~h}$, and the mRNA of $598 I L 7 R, I L 6$ and $T N F$ were measured by using real time qPCR. Relative expression was normalized to internal 599 control $(G A P D H)$ and expressed relative to LPS untreated siControl sample. ${ }^{*} P<0.05$ by paired Student's $t$ 600 test. (j) Three healthy donors' CD127 $7^{\text {high }}$ and $\mathrm{C} 127^{\text {low }}$ monocytes were sorted as in (F), and generation of 601 ATAC-seq data sets were performed for two monocyte populations from each donor. Pie graph shows the 602 percentages of feature open chromatin regions (OCR) in CD127 $7^{\text {high }}$ and C12 $7^{\text {low }}$ monocytes in total OCRs in 603 LPS-treated monocytes by statistical analyses for three ATAC-seq data sets. (k) Three ATAC-seq data sets 604 were combined, and average ATAC-seq signals were calculated in combined ATAC-seq data set for $605 \mathrm{CD} 127^{\text {high }}$ and $\mathrm{C} 127^{\text {low }}$ monocytes around feature OCRs in CD127 $7^{\text {high }}$ monocytes. (l) Motif enrichment 606 analysis in CD127 $7^{\text {high }}$ monocyte featured open chromatin regions. Top 5 most enriched transcription factor 607 binding motifs are shown, and $\mathrm{x}$-axis is $-\log _{10}$ ( $\mathrm{p}$-value) for each enriched motif. Binomial distribution was 608 used for p-value calculation. (m) CD14+ monocytes were pretreated with STAT5 inhibitor $(100 \mu \mathrm{M})$ for $2 \mathrm{~h}$ 609 and then were stimulated with LPS (10 ng/ml). mRNA levels of IL6 (LPS $6 \mathrm{~h}$ ) and TNF (LPS $3 \mathrm{~h}$ ) were 610 measured using qPCR. $* P<0.05$ by paired Student's $t$ test. The results from six independent experiments are 611 shown. 


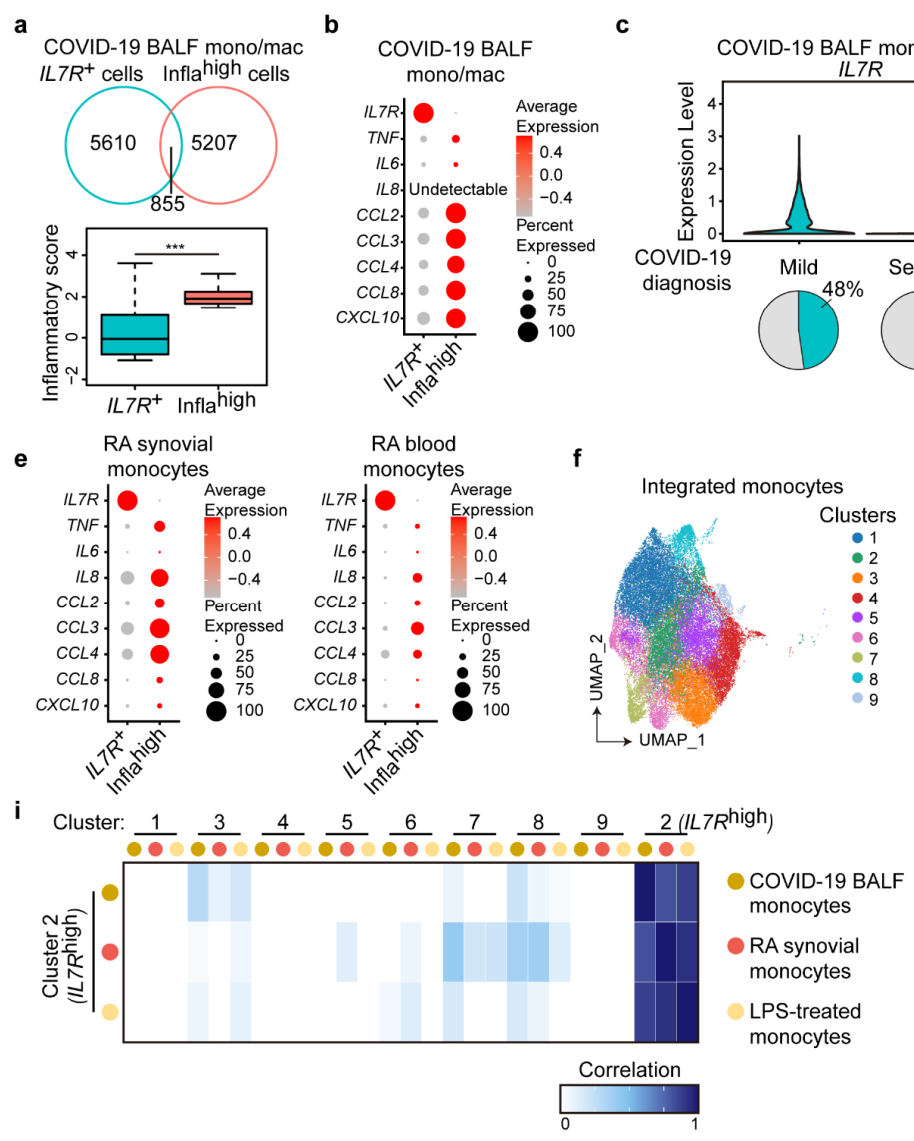

613

614

615

616

617

618

619

620

621

622

623

624

625

626

627

628

629

630

631

\section{Figure 4. CD127 $7^{\text {high }}$ monocytes are anti-inflammatory in human diseases.}

g $\quad L 7 R$
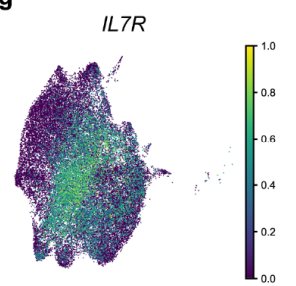

h

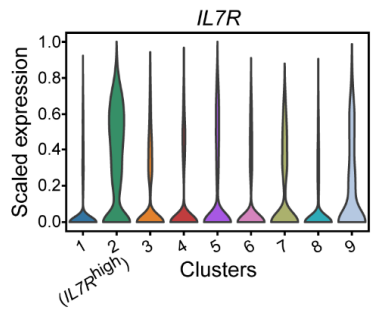

j Top 25 cluster 2 signature genes

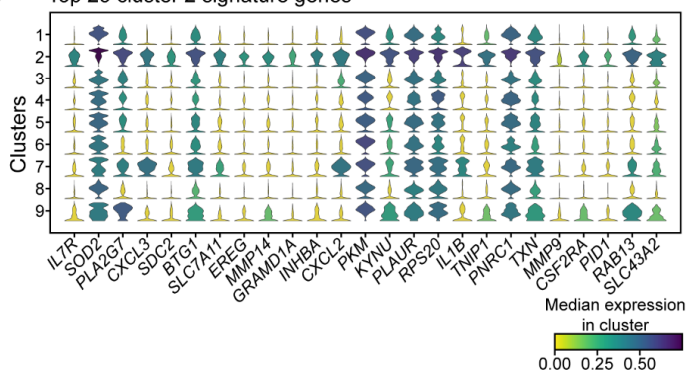

(a, d) Infla ${ }^{\text {high }}$ cells for each disease condition were subgrouped as top $20 \%$ inflammatory cells by inflammatory score in mono/mac from COVID-19 BALF, RA synovial monocytes and RA blood monocytes, respectively. Venn diagrams (upper) show the extent of overlap between $I L 7 R^{+}$cells and inflahigh cells, and box plots (bottom) show the inflammatory score distribution in $I L 7 R^{+}$cells and infla ${ }^{\text {high }}$ cells in mono/mac from COVID-19 BALF (a), RA synovial monocytes and RA blood monocytes (d) as indicated. $* * * P<0.001$ by Wilcoxon rank-sum test. (b, e) Heat maps show the expression of $I L 7 R$ and eight inflammatory genes for inflammatory score calculation in $I L 7 R^{+}$cells and infla ${ }^{\text {high }}$ cells in mono/mac from COVID-19 BALF (B), RA synovial monocytes and RA blood monocytes (e) as indicated. The scaled average expression levels for each gene and percentages of cell expressing each gene in each group were represented by color and size of the corresponding dots, respectively. (c) Mono/mac cells from COVID-19 patients' BALF were subgrouped by the diagnosed disease severity. Violin plot (upper) shows the expression of $I L 7 R$ in BALF mono/mac from mild or severe COVID-19 patients. Pie graphs on the bottom show the percentage of $I L 7 R^{+}$cells in BALF mono/mac from mild or severe COVID-19 patients as indicated. (f) UMAP projection of integrated monocytes from COVID-19 BALF, RA synovial cavity and LPS-treated monocytes. Nine monocyte clusters by unsupervised clustering were indicated by different colors in plot. (g) UMAP projection of integrated monocytes in (f). $I L 7 R$ expression among cells were expressed by color as indicated. (h) Violin plot shows the expression of $I L 7 R$ among nine clusters of integrated-monocytes in (f). Cluster 2 were named as $I L 7 R^{\text {high }}$ 
632 cluster by highest $I L 7 R$ expression among clusters. (i) For populations of COVID-19 BALF monocytes, RA 633 synovial monocytes and LPS-treated monocytes, heat map shows the correlation between cluster 2 cells in 634 each monocytes population and all nine clusters in each monocytes population, respectively. (j) The stacked 635 violin plot shows the expression of top 25 cluster 2 signature genes in all nine monocyte clusters in (f). 636 Median expression levels for each gene in each cluster were expressed by color as indicated. 637 
a

COVID-19 BALF scRNA-seq

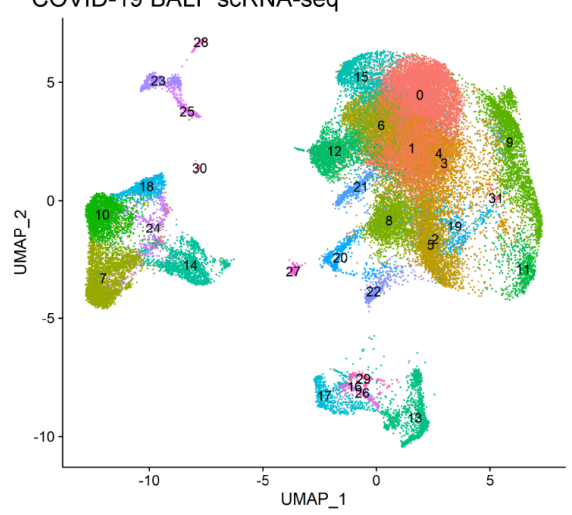

b

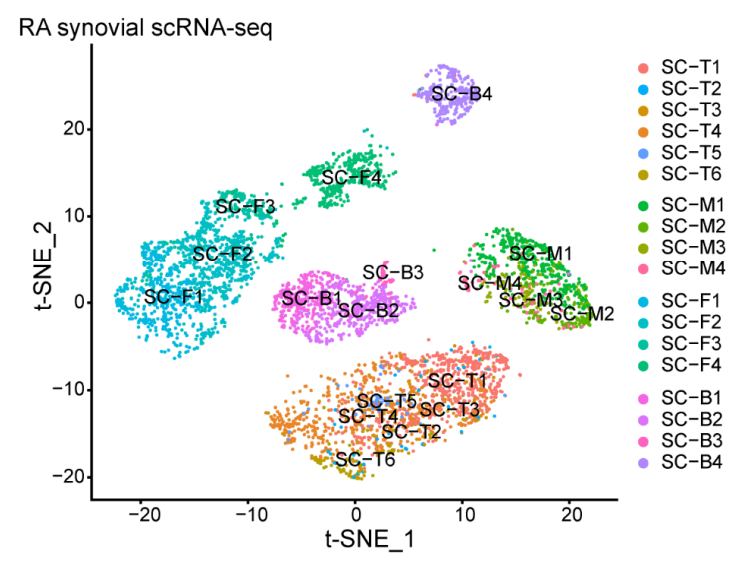

639 Extended Data Figure 1. Clustering analyses of scRNA-seq data sets

640 (a) UMAP projection of broncho-alveolar lavage fluid (BALF) cells from COVID-19 patients. Cell type 641 annotations were labeled for each cluster. Monocyte/macrophage clusters (CD14 ${ }^{\text {high }} \mathrm{CD} 68^{\text {high }}$ ) were used for 642 the subsequent analyses. (b) t-SNE projection of synovial cells from RA patients. Cell type annotations were 643 labeled for each cluster. T, M, F and B represent T cell, monocytes, fibroblasts and B cells, respectively. 644 Monocyte clusters (CD14 $4^{\text {high }}$ ) were used for the subsequent analyses. 
a

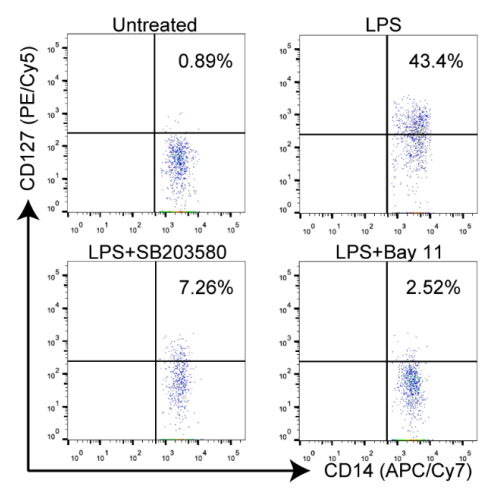

f

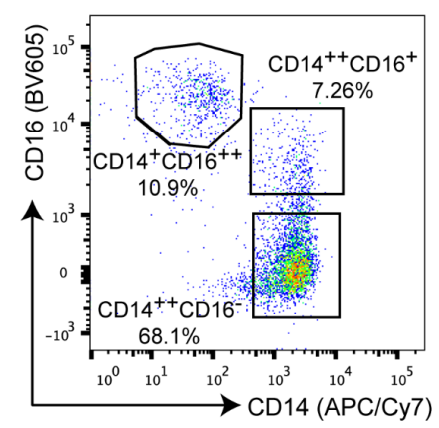

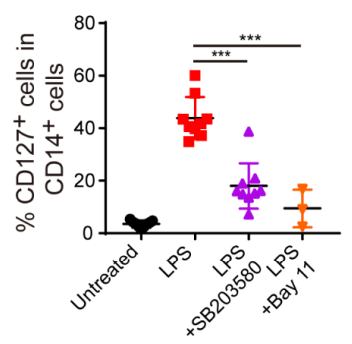

d

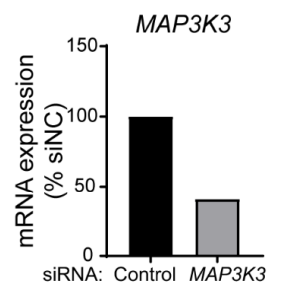

g

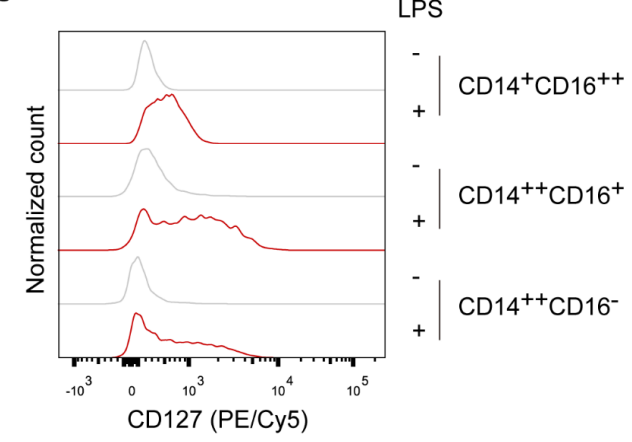

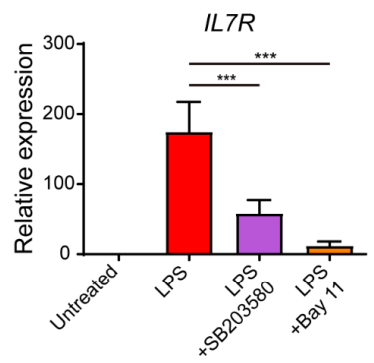

e

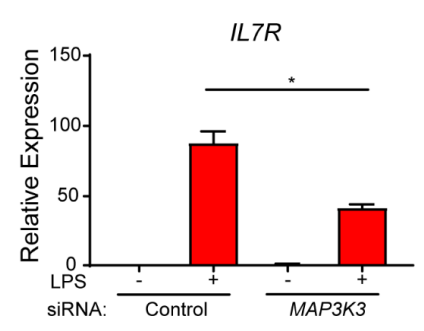

646

647

648

649

650

651

652

\section{Extended Data Figure 2. CD127 upregulation in activated human monocytes is dependent on} canonical TLR signaling and observed in all monocyte subsets.

(a, b) PBMCs form healthy donors were pretreated with DMSO or $10 \mu \mathrm{M}$ SB203580 or $10 \mu \mathrm{M}$ Bay 11-7082 (Bay 11) for $30 \mathrm{~min}$ and subsequently stimulated with or without $10 \mathrm{ng} / \mathrm{ml}$ LPS for 6 hours as indicated. The protein levels of CD127 were measured by flow cytometry and was shown as representative FACS distribution (a) and cumulative percentages (b) in $\mathrm{CD} 14^{+}$monocytes. Each data point represents the result from an independent experiment with cells obtained from a healthy donor. ${ }^{* * *} P<0.001$ by unpaired Student's $t$ test. Data are shown as means \pm SD of multiple independent experiments as listed respectively: Untreated $\mathrm{n}=9$, LPS $\mathrm{n}=9$, SB203580 $\mathrm{n}=9$, Bay 11-7082 $\mathrm{n}=3$. (c) CD14 $4^{+}$monocytes were isolated form healthy donors' PBMCs, and were pretreated with DMSO or $10 \mu \mathrm{M} \mathrm{SB} 203580$ or $10 \mu \mathrm{M}$ Bay 11-7082 (Bay 11) for $30 \mathrm{~min}$ and subsequently stimulated with or without $10 \mathrm{ng} / \mathrm{ml}$ LPS for $6 \mathrm{~h}$ as indicated. The mRNA levels of $I L 7 R$ were measured by qPCR. The relative expression was normalized to internal control $(G A P D H)$ and expressed relative to the untreated sample. $* * * P<0.001$ by unpaired Student's $t$ test. Data are shown as means \pm SD of multiple independent experiments as listed respectively: Untreated $\mathrm{n}=9$, LPS $\mathrm{n}=9$, SB203580 $\mathrm{n}=$ 6, Bay 11-7082 $\mathrm{n}=3$. (d, e) $\mathrm{CD} 14^{+}$monocytes were transfected with negative control or MAP $3 K 3$ specific short interfering RNAs. Two days post transfection, cells were stimulated with LPS ( $10 \mathrm{ng} / \mathrm{ml})$ for $3 \mathrm{~h}$. Knock down efficiency of MAP $3 K 3$ was examined (d), and mRNA induction of $I L 7 R$ by LPS stimulation in siControl and siMAP3K3 transfected cells was measured by qPCR (e). Relative expression was normalized 
665 to internal control $(G A P D H)$ and expressed relative to LPS untreated siControl sample. $* P<0.05$ by paired 666 Student's $t$ test. $I L 7 R$ expression data are shown as means \pm SD of three independent experiments with cells 667 from one healthy donor for each data set. (f, $\mathbf{g})$ Three monocyte populations were gated by CD14 and CD16 668 expression in flow cytometry analysis of human PBMCs. CD127 was also stained in PBMCs with or without 669 LPS stimulation, and CD127 upregulation in three human monocyte populations was analyzed by gating 670 strategy shown in (f). Representative FACS distribution (g, left) and mean fluorescence intensity (MFI) (g, 671 right) for CD127 expression are shown. 
a
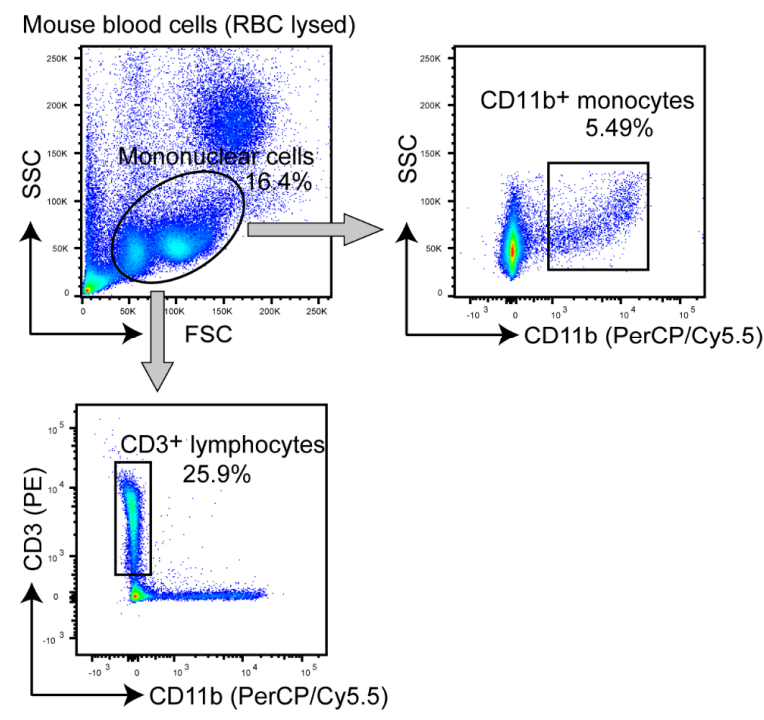

d

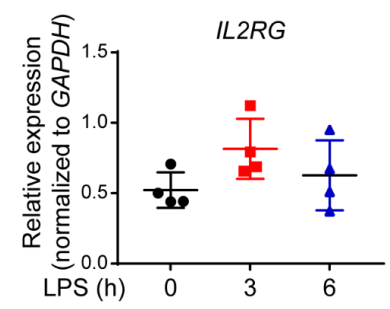

Homeostatic mouse $\mathrm{CD}_{11 b^{+}}$monocytes

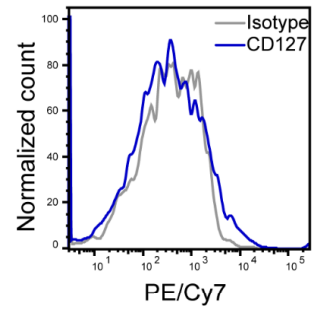

C

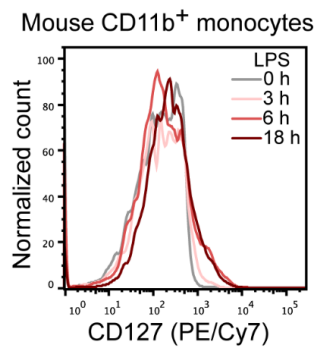

Homeostatic mouse $\mathrm{CD}^{+}$lymphocytes
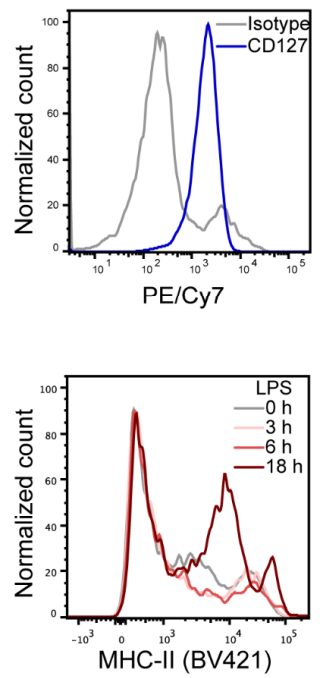

Extended Data Figure 3. Human but not mouse activated monocytes are CD127 positive and functionally competent for IL-7 receptor signaling.

(a) Gating strategy for $\mathrm{CD} 11 \mathrm{~b}^{+}$mouse monocytes and $\mathrm{CD}^{+}$mouse lymphocytes from red blood cell (RBC)

。

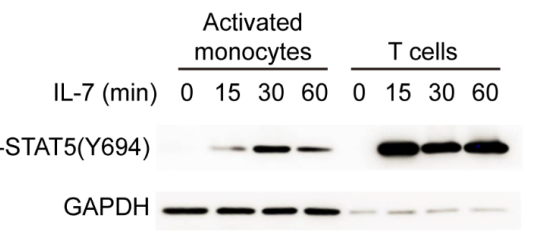
lysed mouse peripheral blood cells. (b, c) Mouse blood cells (RBC lysed) were treated with or without 100 ng/ml LPS for different time points as indicated. The expression of CD127 (b) and MHC-II (c) on mouse

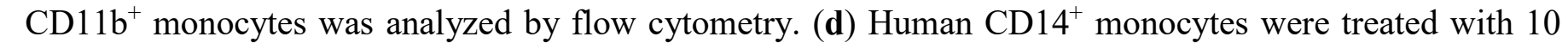
$\mathrm{ng} / \mathrm{ml}$ LPS for $3 \mathrm{~h}$ and $6 \mathrm{~h}$, the mRNA levels of $I L 2 R G$ were measured by qPCR. Each data point represents the result from an independent experiment with cells obtained from a healthy donor. Relative expression was normalized to $G A P D H$ as internal control. Data are shown as means \pm SD of four independent experiments. (e) Human $\mathrm{CD}_{14}^{+}$monocytes were pretreated with $10 \mathrm{ng} / \mathrm{ml}$ LPS for $6 \mathrm{~h}$, and subsequently stimulated with recombinant human IL-7 $(10 \mathrm{ng} / \mathrm{ml})$ for the indicated time. Meanwhile, $\mathrm{CD}^{+} \mathrm{T}$ cells from the same donor were treated with $10 \mathrm{ng} / \mathrm{ml}$ IL-7 for the indicated time. The protein levels of p-STAT5(Y694) were detected by western blotting. GAPDH was used as a loading control. 
a

ATAC-seq signals in CD12 $7^{\text {high }}$ monocyte feature OCRs $(n=327)$

CD127 $\frac{\text { Donor } 1}{\text { High Low }} \frac{\text { Donor } 2}{\text { High Low }} \frac{\text { Donor } 3}{\text { High Low }}$

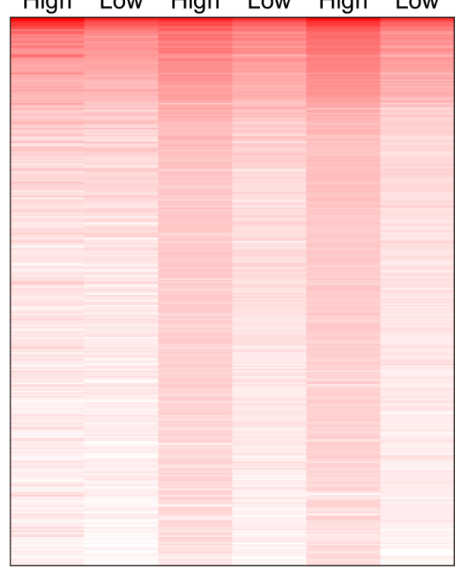

b

Feature OCRs in $\mathrm{CD} 127^{\text {high }}$ monocytes

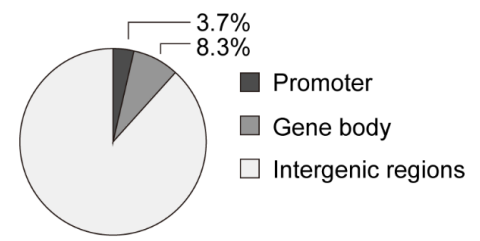

C

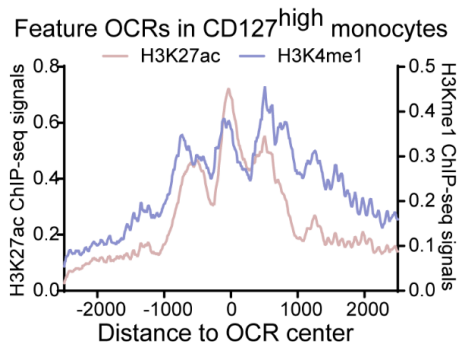

689 Extended Data Figure 4. Open chromatin regions (OCRs) in CD127 ${ }^{\text {high }}$ monocytes display enhancer690 like features.

691 (a) Heat map shows the ATAC-seq signals in OCRs featured in CD127 $7^{\text {high }}$ monocytes and in CD127 low 692 monocytes from three independent experiments with three blood donors. The ATAC-seq reads count was 693 normalized (per 10 million mapped reads) and then $\log _{10}(n+1)$ transformed for expression in heat map. (b) 694 Pie graph shows the genomic distribution of CD127 $7^{\text {high }}$ monocyte-featured OCRs. (c) Average ChIP-seq 695 signals of H3K27ac and H3K4me1 in LPS activated monocytes were assessed around CD127 ${ }^{\text {high }}$ monocyte696 featured OCRs and expressed by different colors as indicated in the plot. 
698

699

700

701

702

703

704

705

706

707

708

709

710

711

712

713

714

715 a
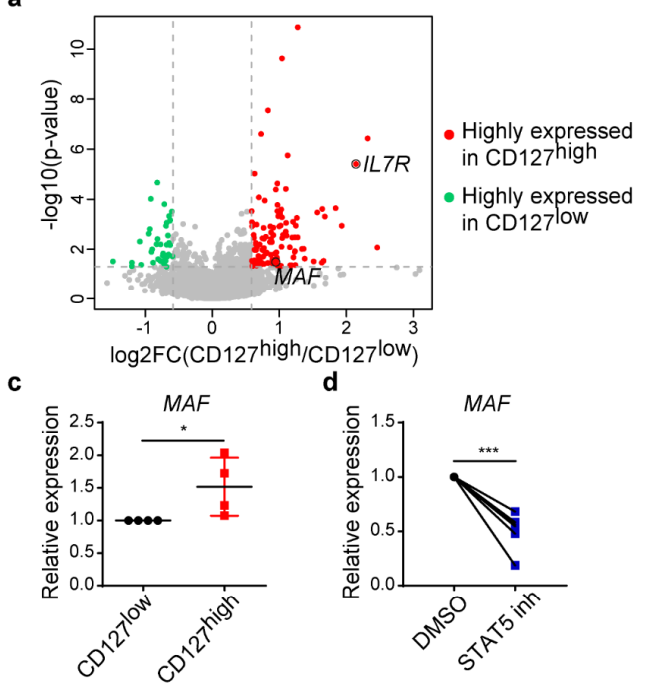

e
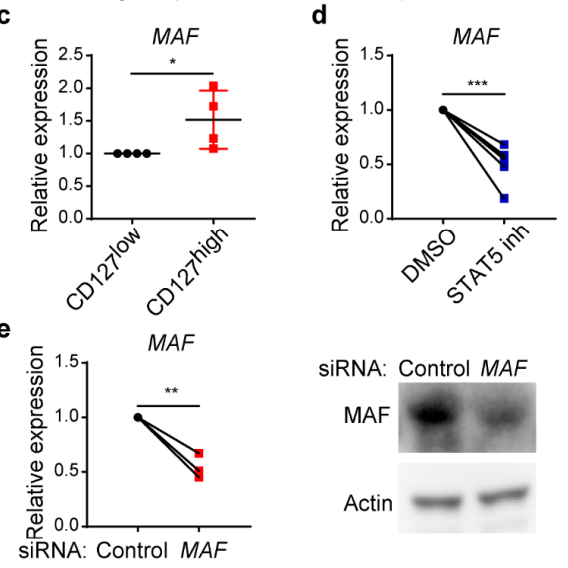

f

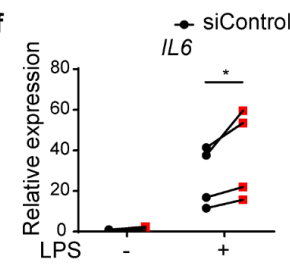

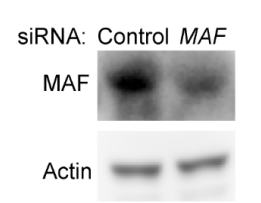

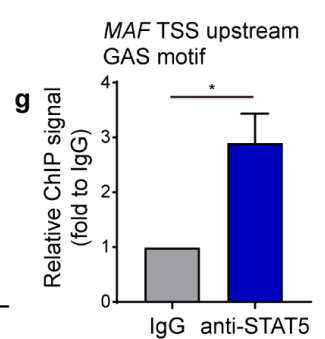

b

Highly expressed genes in CD127high Expression by RNA-seq (FPKM)

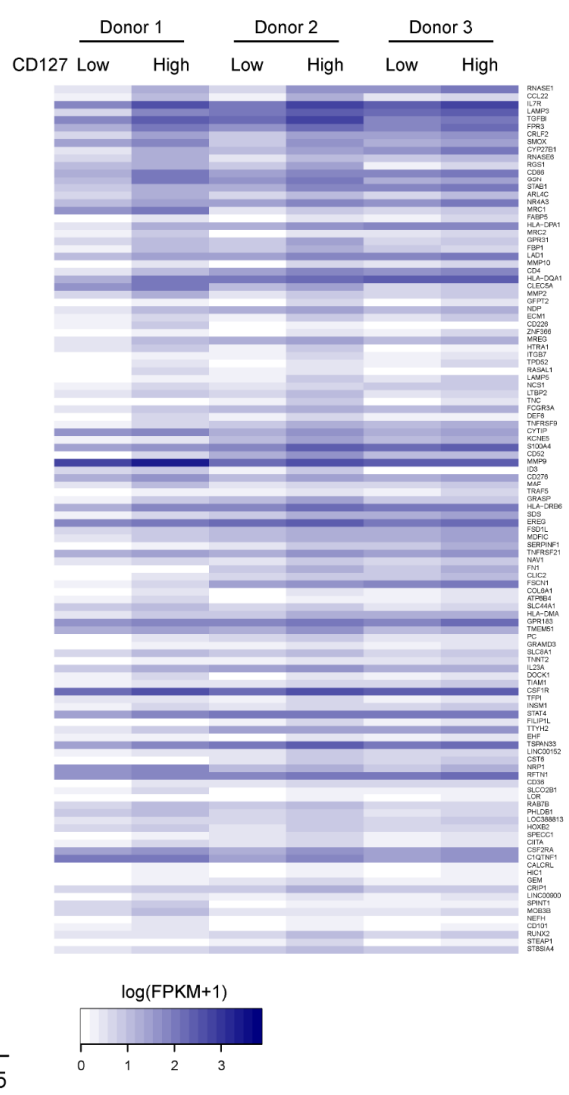

Expression fold change
$\log 2\left(\mathrm{CD} 127^{\text {high }} / \mathrm{CD} 127^{\text {low }}\right)$

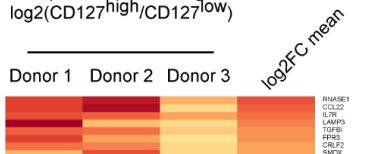

\section{Extended Data Figure 5. CD127-STAT5-c-Maf axis imposes an anti-inflammatory loop in activated} monocytes

(a) Volcano plot shows the differentially expressed genes by RNA-seq between CD127 $7^{\text {high }}$ and CD127 $7^{\text {low }}$ populations from $\mathrm{CD}_{1} 4^{+}$monocytes upon $6 \mathrm{~h}$ LPS stimulation. Red colors indicate highly expressed genes in $\mathrm{CD} 127^{\text {high }}$ population, and green colors indicate highly expressed genes in CD127 $7^{\text {low }}$ population. Two CD127 $7^{\text {high }}$ highly expressed genes, $I L 7 R$ and $M A F$, were highlighted with black circles. The dash lines indicate the threshold of $p$-value ( $p$-value $<0.05)$ and fold change $(\geq 1.5$ fold for upregulation and $\leq 0.67$ fold for downregulation) for identifying differentially expressed genes. Fold changes are shown as mean value of $\mathrm{CD} 127^{\text {high }} \mathrm{CD} 127^{\text {low }}$ ratio from three healthy donors. (b) Heat map shows the highly expressed genes in $\mathrm{CD} 127^{\text {high }}$ monocytes in (A). The original expression ( $\log _{10}$ transformed FPKM +1 , left) and expression fold change ( $\log _{2}$ transformed CD127 $7^{\text {high }} / \mathrm{CD} 127^{\text {low }}$, right) were shown. Fold changes are shown as the individual value in each donor and the mean value from three donors. (c) The mRNA expression of MAF in CD127 high and $\mathrm{CD} 127^{\text {low }}$ monocytes was measured by qPCR. Relative expression was normalized to internal control $(G A P D H)$ and expressed relative to $\mathrm{CD} 127^{\text {low }}$ sample. Each data point represents the result from an independent experiment with cells obtained from a healthy donor. ${ }^{*} P<0.05$ by paired Student's $t$ test. Data are shown as means \pm SD of four independent experiments. (d) CD14 monocytes were pretreated with STAT5 inhibitor $(100 \mu \mathrm{M})$ for $2 \mathrm{~h}$ and then were stimulated with LPS $(10 \mathrm{ng} / \mathrm{ml})$ for $6 \mathrm{~h}$. mRNA of $M A F$ 
716 was measured by qPCR. Relative expression is normalized to internal control (GAPDH) and expressed 717 relative to DMSO treated sample. Each data point represents the result from an independent experiment with 718 cells obtained from a healthy donor. ${ }^{* * *} P<0.001$ by paired Student's $t$ test. The results from six independent 719 experiments are shown. (e, f) $\mathrm{CD} 14^{+}$monocytes were transfected with negative control or $M A F$ specific short 720 interfering RNA (siControl or si $M A F$ ). Two days post transfection, knockdown efficiency was assessed by 721 measuring $M A F$ mRNA with qPCR (e left, relative expression is normalized to internal control (GAPDH) 722 and expressed relative to siControl group) and MAF protein levels with immunoblotting (e right). Two days 723 post transfection, cells were stimulated with LPS $(10 \mathrm{ng} / \mathrm{ml})$ for $3 \mathrm{~h}$ and mRNA levels of IL6 and TNF were 724 measured by qPCR (f). Relative expression was normalized to internal control (GAPDH) and expressed 725 relative to LPS untreated siControl sample. Each data point represents the result from an independent 726 experiment with cells obtained from a healthy donor. ${ }^{*} P<0.05, * * P<0.01$ by paired Student's $t$ test. The 727 results from four independent experiments are shown. (g) Occupancy of STAT5 on the MAF transcription 728 start site (TSS) upstream GAS motif was assessed by ChIP-qPCR in THP-1 cells with 6 h LPS stimulation 729 prior to $30 \mathrm{~min}$ IL-7 stimulation. IgG ChIP was used as negative control, and relative STAT5 ChIP signals 730 were expressed relative to IgG ChIP control sample. $* P<0.05$ by paired Student's $t$ test. Data are shown as 731 means \pm SD of three independent experiments. 
733

734

735

736

737

738

739

740

741

742

743

744

745

746

747

748

749

750 COVID-19 BALF mono/mac

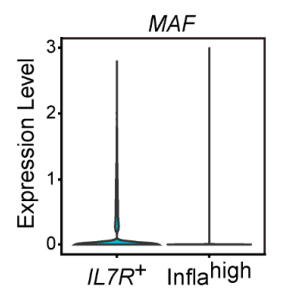

b

COVID-19 BALF lymphoid cells

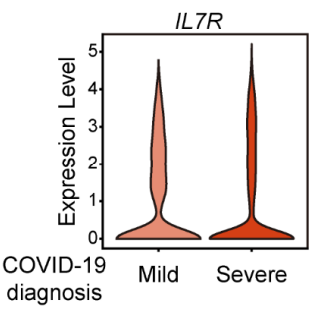

e

Integrated monocytes/macrophages
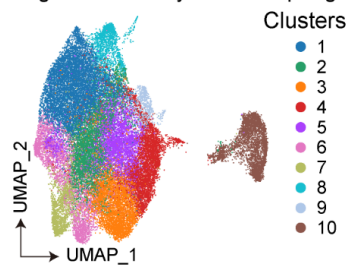

c

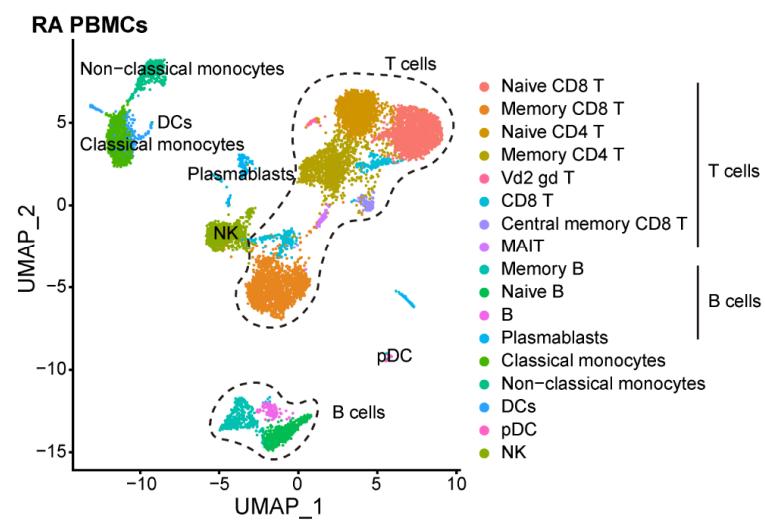

f

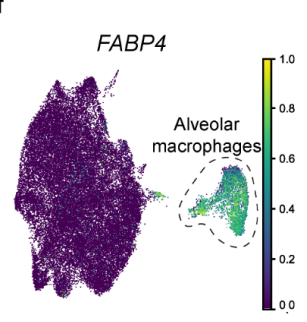

g

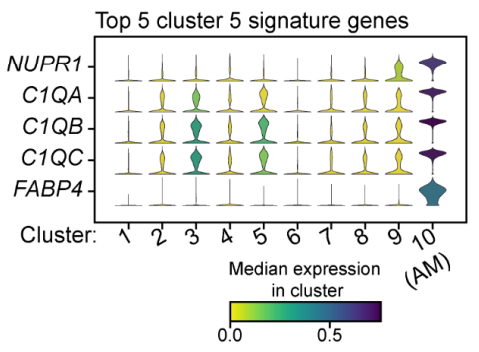

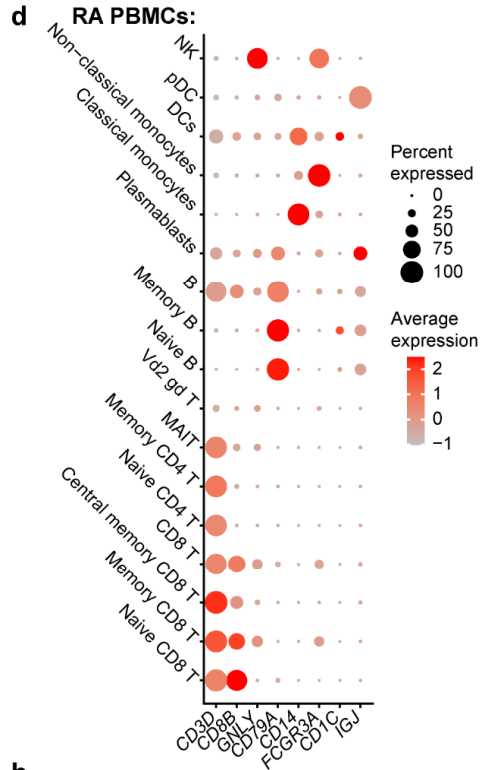

h

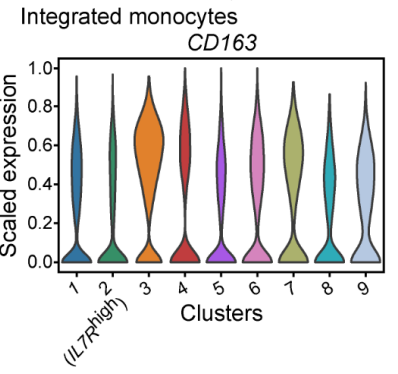

\section{Extended Data Figure 6. CD127 expression designates a functionally distinct monocyte subset}

(a) Violin plot shows the $M A F$ expression distribution in $I L 7 R^{+}$cells and inflah cells in monocytes/macrophages from COVID-19 patients' BALF in Fig. 4a. (b) Lymphoid cells from COVID-19 patients' BALF were subgrouped by the diagnosed disease severity. Violin plot shows the expression of $I L 7 R$ in BALF lymphoid cells from mild or severe COVID-19 patients. (c) UMAP projection of PBMCs from RA patient. Cell type annotations were labeled for each cluster. (d) Heat map shows the expression of hallmark genes in different cell clusters from RA PBMCs. The scaled average expression levels of marker genes and percentage of cell expressing marker genes were expressed by color and size of each dot corresponding to cell clusters, respectively. (e) UMAP projection of integrated monocytes/macrophages from COVID-19 BALF, RA synovial cavity and LPS-activated monocytes. Ten clusters by unsupervised clustering were indicated by different colors in plot. (f) UMAP projection of integrated monocytes/macrophages in (e). $F A B P 4$ expression in cells was quantitatively visualized by the indicated colors. Cells corresponding to Cluster 5 were highlighted by dotted line as alveolar macrophages given the specific FABP4 expression pattern. (g) The stacked violin plot shows the expression of top 5 cluster 5 signature genes in all ten clusters shown in (e). Median expression levels for each gene in each cluster were expressed by colors as indicated. (h) Violin plot shows the expression of CD163 among nine clusters of integrated monocytes in Fig. 4f, in which cluster 2 was designated as $I L 7 R^{\text {high }}$ cluster. 


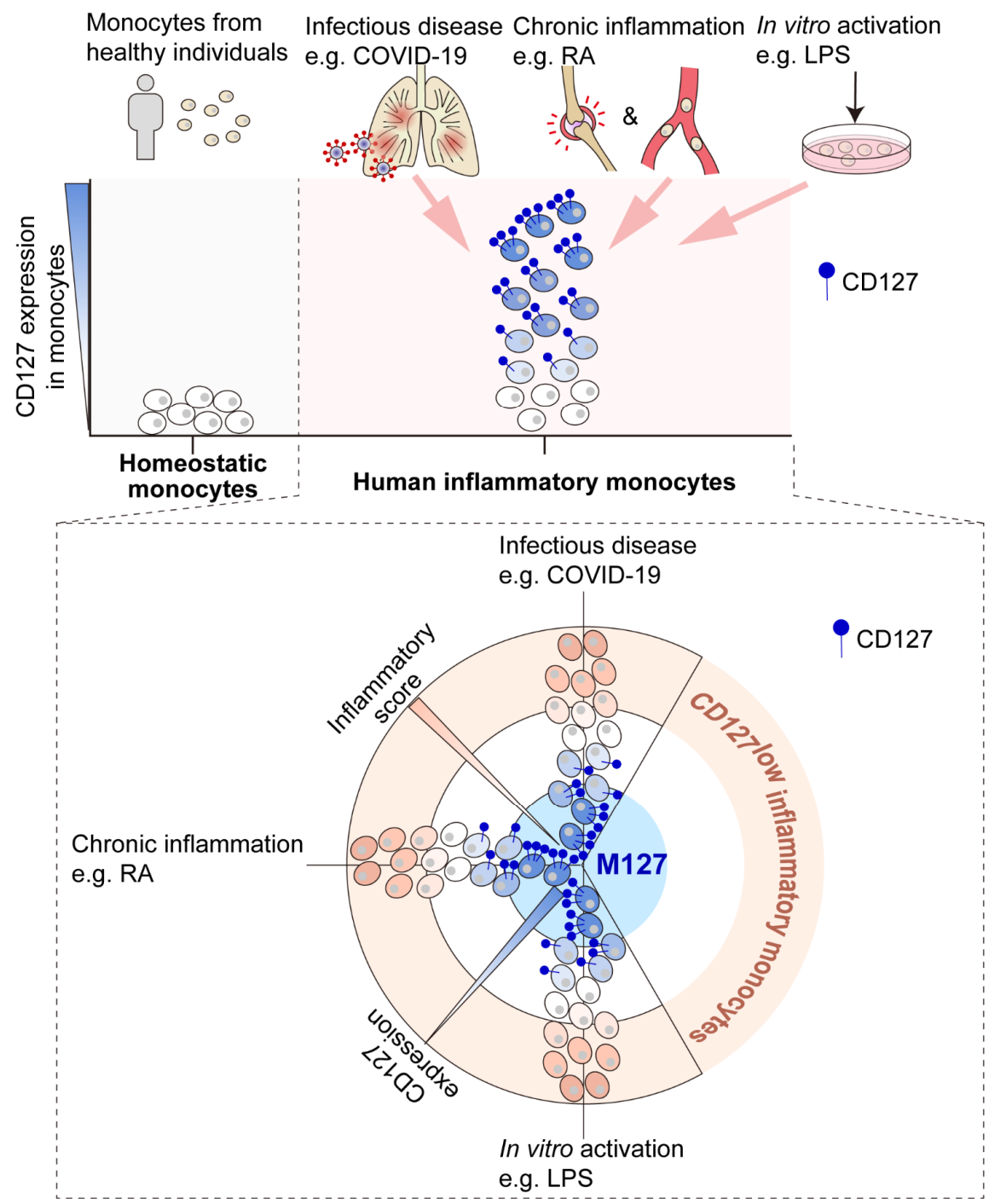

Extended Data Figure 7. M127 represents a unique hypo-inflammatory monocyte population in

\section{human inflammatory diseases}

754 CD127 is minimally expressed on homeostatic monocytes from healthy individuals. Upregulation of CD127

755 is a unified hallmark event for human monocyte activation under various inflammatory conditions including 756 COVID-19, RA and stimulated blood monocytes. CD127 $7^{+}$inflammatory monocytes, M127, display unique 757 gene signatures and subdued inflammatory phenotypes imposed by CD127-STAT5 signaling. As a result, 758 CD127 levels inversely correlate with the inflammatory capacity of human monocytes. Identification and 759 characterization of M127 highlight the previously underappreciated functional diversity among human monocytes in inflammatory diseases. 
761 Extended Data Table 1. qPCR primers

\begin{tabular}{|l|l|l|}
\hline Gene & Forward $\left(5^{\prime}--3^{\prime}\right)$ & Reverse $\left(5^{\prime}--3^{\prime}\right)$ \\
\hline$I L 7 R$ & TCCAACCGGCAGCAATGTAT & TGACCAACAGAGCGACAGAG \\
\hline$I L 2 R G$ & GTGCAGCCACTATCTATTCTCTG & GTGAAGTGTTAGGTTCTCTGGAG \\
\hline$I L 6$ & ACCCCCAATAAATATAGGACTGGA & TTCTCTTTCGTTCCCGGTGG \\
\hline$T N F$ & CCTCTCTCTAATCAGCCCTCTG & GAGGACCTGGGAGTAGATGAG \\
\hline$M A P 3 K 3$ & ACCAGCATCAACAGTGAGGG & TGATTTCCGGAAGGATGGGC \\
\hline$M A F$ & ACTGGCAATGAGCAACTCCG & CACTGGCTGATGATGCGGTC \\
\hline
\end{tabular}


764 Extended Data Table 2. Antibodies used for the experiments

\begin{tabular}{|c|c|c|}
\hline Antibodies & Manufacturer & Catalog number \\
\hline Anti-CD68 antibody & Abcam & ab201340 \\
\hline Anti-human CD127 antibody & Invitrogen & PA5-97870 \\
\hline $\begin{array}{l}\text { Goat anti-rabbit IgG (H\&L)-HRP } \\
\text { conjugated }\end{array}$ & Bioeasy (Beijing) Technology CO. Ltd & BE0101 \\
\hline $\begin{array}{l}\text { Goat anti-mouse IgG (H\&L)-HRP } \\
\text { conjugated }\end{array}$ & Bioeasy (Beijing) Technology CO. Ltd & BE0102 \\
\hline Alexa Fluor $^{\mathrm{TM}} 488$ goat anti-mouse IgG & Life Technologies & B40941 \\
\hline Alexa Fluor ${ }^{\mathrm{TM}} 555$ goat anti-rabbit $\operatorname{IgG}$ & Invitrogen & A27039 \\
\hline Anti-mouse $\mathrm{CD} 3 \varepsilon$ antibody PE & Biolegend & 100307 \\
\hline Anti-mouse CD11b PerCP-Cyanine5.5 & eBioscience & $45-0112-82$ \\
\hline Anti-mouse CD127 PE-Cyanine7 & Biolegend & 135013 \\
\hline $\begin{array}{l}\text { PE-Cy7 Rat IgG2a, } \kappa \text { isotype control } \\
\text { antibody }\end{array}$ & Biolegend & 400521 \\
\hline Anti-mouse MHC class II BV421 & eBioscience & $48-5321-82$ \\
\hline $\begin{array}{l}\text { Anti-human CD127 antibody PE- } \\
\text { Cyanine5 }\end{array}$ & Biolegend & 351323 \\
\hline $\begin{array}{l}\text { Anti-human CD14 antibody APC- } \\
\text { Cyanine } 7\end{array}$ & Biolegend & 301820 \\
\hline Anti-human IL-6 antibody PE & Biolegend & 501106 \\
\hline Anti-human TNF- $\alpha$ antibody PE & Biolegend & 502908 \\
\hline $\begin{array}{l}\text { PE Rat IgG2b, } \kappa \text { isotype control } \\
\text { antibody }\end{array}$ & Biolegend & 400607 \\
\hline Anti-human CD16 antibody BV605 & Biolegend & 302039 \\
\hline $\begin{array}{l}\text { Anti-GAPDH Mouse Monoclonal } \\
\text { Antibody }\end{array}$ & Bioeasy (Beijing) Technology CO. Ltd & BE0023 \\
\hline $\begin{array}{l}\text { Anti- } \beta \text {-Actin Mouse Monoclonal } \\
\text { Antibody }\end{array}$ & Abclonal & $\mathrm{AC} 026$ \\
\hline $\begin{array}{l}\text { Anti-Phospho-Stat5 (Tyr694) Rabbit } \\
\text { Monoclonal Antibody }\end{array}$ & Cell Signaling Technology & $9314 \mathrm{~S}$ \\
\hline Anti-Stat5 Antibody & Cell Signaling Technology & $9363 \mathrm{~S}$ \\
\hline Anti-c-Maf Antibody & Santa Cruz Biotechnology & sc-518062 \\
\hline
\end{tabular}

